grand prématuré. Sans réalisation du test sudoral, seule l'analyse moléculaire permet de poser le diagnostic. Quand on met en évidence chez le nouveau-né décédé 2 mutations $\mathrm{CF}$, l'incertitude quant à la présence de la mucoviscidose est aisément levée et toute grossesse ultérieure est facilement gérable par le conseil génétique. Il en est tout autrement quand l'analyse moléculaire révèle des anomalies du gène CFTR discutables en termes de pathogénicité. Nous rapportons ici le cas d'un nouveau-né grand prématuré ( $26 \mathrm{SA}+3$ jours) décédé à J13 dans un contexte de défaillance multi-viscérale due à une entérocolite ulcéro-nécrosante et suspect de mucoviscidose en raison d'une TIR J3 élevée et de la présence d'une hétérozygotie pour la mutation $\mathrm{CF}$ connue $2183 \mathrm{AA}>\mathrm{G}$ d'origine maternelle. L'analyse du père du nouveau-né a montré la présence (1) d'anomalies génétiques connues, rapportées comme peu pathogènes mais surtout (2) d'un faux sens (C1355Y, exon 22), non décrit à ce jour et que l'analyse par plusieurs logiciels de prédiction qualifie de probablement délétère avec des scores de pathogénicité très élevés. Par la suite, la présence de ce faux sens d'origine paternelle a été retrouvée chez le nouveau-né décédé. La description de cette mutation chez ce grand prématuré renforce d'une part la suspicion de mucoviscidose sans toutefois faire avec certitude de cette maladie la cause du décès et peut d'autre part aider le conseil génétique de ce couple en vue d'un nouveau projet parental. Des études fonctionnelles de ce faux sens devraient permettre de confirmer son statut de mutation CF.

Mots-clés : mucoviscidose, décès néonatal, mutation C1355Y.

口P651. ÉTIOLOGIES DES HYPERECHOGÉNICITÉS INTESTINALES FETALES ET DEVENIR PÉDIATRIQUE À COURT TERME. À PROPOS DE 99 CAS PRIS EN CHARGE AU CHRU DE LILLE DE 2004 À 2007

G. Debaecker-Peulmeule (1), F. Petit (2), S. Petit (3), L. Devisme (3), S. Manouvrier-Hanu (2), M. Holder-Espinasse (2)

(1) Service de néonatologie, Hôpital Jeanne-de-Flandre, CHRU Lille, France; (2) Service de génétique clinique, Hôpital Jeanne-de-Flandre, CHRU Lille, France; (3) Service d'anatomo-pathologie, Centre de biologie pathologie, CHRU Lille, France

Contact : m-holder@chru-lille.fr

Objectifs : Une hyperéchogénicité intestinale fœetale (HEI) est découverte dans 0,2 à $1,8 \%$ des grossesses. La signification clinique de ce signe échographique n'est pas encore clairement établie à ce jour. Il peut être un variant de la normale au second trimestre de grossesse, ou, même isolé, être associe à une évolution défavorable de la grossesse. Cette étude rétrospective a permis de connaître l'issue de grossesses pour lesquelles une hyperéchogénicité intestinale fœtale isolée ou associée à d'autres anomalies échographiques avait été à l'origine d'un bilan complémentaire. Méthodes : Quatre vingt dix neuf suivis et issues de grossesses ont été étudiés entre 2004 et 2007, à la maternité Jeanne-de-Flandre du CHRU de Lille. Résultats : Les enfants étaient en bonne santé apparente à 2 ans de vie dans $59 \%$ des cas. L'issue de la grossesse était défavorable dans $32 \%$ des cas, correspondant à 21 interruptions médicales de grossesses (IMG), 8 morts fœtales in utero et 3 décès avant 6 mois de vie (une prématurité, un volvulus digestif, un syndrome polymalformatif). Les indications d'IMG regroupaient 5 pathologies maternoplacentaires, 5 mucoviscidoses, 4 anomalies chromosomiques, 2 infections: CMV et rubéole, 5 syndromes poly malformatifs. Il n'existait aucun antécédent familial chez les foetus atteints de mucoviscidose. Dix-sept enfants étaient nés prématurés avec un âge gestationnel moyen de 33 semaines d'aménorrhée. Un retard de croissance intra-utérin était observé dans $28 \%$ des cas. Parmi les 66 enfants nés vivants, 7 présentaient des pathologies : 2 syndromes d'apple-peel, une mucoviscidose, une maladie de Steinert, un syndrome polymalformatif, un syndrome VACTERL et une dilatation pyélocalicielle. Conclusion: Devant une HEI, même isolée, il parait indispensable de proposer un bilan minimum comprenant un caryotype, une recherche de mucoviscidose, un bilan infectieux ainsi qu'un suivi échographique et une surveillance périnatale. Les parents peuvent, à priori, être rassurés lorsque le bilan complémentaire est négatif et qu'aucune étiologie n'est retrouvée pour expliquer l'HEI isolée.

Mots-clés: hyperéchogénicité intestinale, mucoviscidose, syndromes malformatifs.

\section{Prise en charge des maladies génétiques : du conseil génétique au traitement}

\section{P652. CREATION D'UNE NEWSLETTER DU CONSEILLER EN GÉNÉTIQUE}

S. Aissaoui $(1,2,3)$, N. Pasz $(1,4)$, E. Consolino $(1,5)$, E. Chipoulet $(1$, $6,7)$, M.A. Voelckel $(1,8)$, C. Cordier $(1,9,10)$
(1) Association Française des Conseillers en Génétique, Marseille, France: (2) Service de Génétique Moléculaire et Médicale, Hôpital Édouard-Herriot, Lyon, France; (3) Unité Clinique d'Oncologie Génétique, Centre Léon-Bérard, Lyon, France; (4) Service de Génétique clinique Guy Fontaine, CHRU Hôpital Jeanne-de-Flandre, Lille, France; (5) Service de Génétique Oncologique, Institut Gustave Roussy, Villejuif, France ; (6) Service d'Oncogénétique, Institut Claudius Regaud, Toulouse, France; (7) Service d'Oncologie Digestive, CHU de Purpan, Toulouse, France: (8) Département de Génétique Médicale, Hôpital D'Enfants de la Timone, Marseille; (9) Service d'Oncogénétique, Institut Claudius Regaud, Toulouse, France; (10) Service d'Oncologie Digestive, CHU de Purpan, Toulouse, France

Contact : souria.aissaoui@chu-lyon.fr

L'Association Française des Conseillers en Génétique, AFCG, a été créée en 2005 (JO du 18 juin 2005) dès la première année de formation des nouveaux professionnels de santé que sont les conseillers en génétique. L'AFCG a pour but non seulement de promouvoir ce nouveau métier mais également d'informer ses membres de l'évolution de la profession. Ainsi, afin d'apporter une dynamique supplémentaire au site internet de l'association, cinq bénévoles rédigent une lettre d'information électronique trimestrielle, la «newsletter » de l'AFCG. Y sont mentionnés les informations générales sur les démarches administratives, les questions d'actualité, l'étude de publications, les congrès et réunions de travail à venir ainsi que les postes à pourvoir. L'équipe en charge de cette mission est composée d'un rédacteur en chef, une secrétaire chargée de communication, une chargée d'actualités, une chargée de relations ainsi que d'une chargée d'études. La création de ce moyen d'information montre l'engouement de ces professionnels pour leur métier, leur volonté de s'impliquer pleinement dans sa reconnaissance et de contribuer à son évolution. La première newsletter, sortie en Mars 2009, a été distribuée, grâce à une mailing liste, à la totalité des membres de l'AFCG (92 à ce jour). Elle est disponible sur le site. Monsieur le Professeur Jean-François MATTEI, ancien Ministre de la Santé, Président de la Croix Rouge Française, nous a fait l'honneur d'inaugurer ce document en rédigeant l'éditorial. Il y fait part de la « nécessité des conseillers en génétique ».

Mots-clés : newsletter, information, conseil génétique.

- P53. POUR LA CRÉATION DE RELAIS ENTRES LES ASSO. CIATIONS DE PATIENTS ATTEINTS DE MALADIE GÉNÉTIQUE ET LES CONSEILLERS EN GÉNÉTIQUE

N. Pasz (1, 2), S. Devaux (3, 2), B. Troude $(4,2)$, L. Monteil $(5,2)$, M.A. Voelckel $(6,2)$

(1) Service de Génétique Clinique, CHRU de Lille, France ; (2) Association Française des Conseillers en Génétique, France; (3) Services de Génétique Oncologique et d'Urologie, CHU de Bicêtre, Hôpital Necker. France; (4) Service de Génétique Médicale, CHU de Clermont-Ferrand, France: (5) Service de Génétique Médicale, Hôpital PURPAN, CHU de Toulouse, France; (6) Département de Génétique Médicale, APH Marseille, France

Contact:n-pasz@chru-lille.fr

L'Association Française des Conseillers en Génétique (AFCG), créée en mai 2005, compte parmi ses nombreux objectifs l'information sur les maladies génétiques et la médecine prédictive, sous toutes ses formes, à l'attention des médecins, des professionnels de santé, des familles, du public et des associations de patients. Une commission de l'AFCG baptisée « Relais avec les associations $»$ existe depuis 2007. Les desseins de cette commission se sont progressivement développés autour de différents points. Tout d'abord, celui de l'amélioration et de l'optimisation de la prise en charge des patients atteints de maladie génétique dans leur parcours de soin, puis celui d'une meilleure compréhension de la part des patients et de leur famille par une vulgarisation du langage médical, et enfin celui d'une information et d'une sensibilisation du grand public, toujours en collaboration avec les associations. Des actions ont déjà été menées dans ce sens, que ce soit par la collaboration entre l'AFCG et Orphanet (participations à l'Encyclopédie Grand Public, au forum Internet Orphanet en 2006), par la présence de certains membres de l'AFCG à des réunions d'information d'associations de patients (Association du Syndrome de Prader Willi, Association HNPCC France, etc.), ou encore par des interventions lors de l'émission télévisée Téléthon ( Paroles de nuit » en 2005, « Marche des maladies rares » en 2007 et 2008). Le but de notre étude est d'évaluer les attentes et les besoins des associations de malades et de leur famille pour pouvoir mener des actions en conséquence. Pour réaliser ce projet, nous sommes intervenus lors d'une réunion d'information des membres de l'Alliance Maladies Rares en mai 2009 afin de sensibiliser un maximum d'associations. Cette intervention nous a permis, non seulement de présenter et de promouvoir notre métier, mais aussi de répondre à un certain 
nombre d'interrogations et de recevoir des commentaires en faveur d'une collaboration plus active entre les associations et les conseillers en génétique. L'intervention s'est terminée par la distribution d'une lettre d'information et d'un questionnaire. D'autres associations, qui n'étaient pas présentes à cette journée, ont été sollicitées en recevant les mêmes documents par message électronique. Nous rapporterons les résultats du recueil et de I'analyse de ces questionnaires afin de planifier des actions efficaces répondant précisément à leurs attentes.

Mots-clés : conseillers en génétique, association de malades, relais.

口P654. LE CONSEIL GÉNÉTIQUE APRÈS DÉPISTAGE NÉONATAL DE LA MUCOVISCIDOSE EN LANGUEDOCROUSSILLON

E. Haquet (1), F. Counil (2), K. Baudry (1), J. Puechberty (1), P. Sarda (1), C. Coubes (1)

(1) Service de génétique médicale, Hôpital Arnaud-de-Villeneuve, Montpellier, France ; (2) Centre de Ressources et de Compétences de la Mucoviscidose, Hôpital Arnaud-de-Villeneuve, Montpellier, France

Contact : e-haquet@chu-montpellier.fr

Le dépistage néonatal systématique de la mucoviscidose a été mis en place en France depuis 2002. Il associe un dosage de la trypsine immunoréactive (TIR) à une analyse en biologie moléculaire de l' $\mathrm{ADN}$ du patient si le dosage est élevé. Les enfants ainsi dépistés sont invités à consulter au Centre de Ressources et de Compétences de la Mucoviscidose (CRCM), afin que soient pratiqués des examens complémentaires (test de la sueur...) et de poser ou non le diagnostic de mucoviscidose. Pour les patients « faux positifs » (chez qui la mucoviscidose n'a pas été confirmée), seules les mutations fréquentes sont recherchées. Pour les enfants malades, le laboratoire s'attache à rechercher les deux mutations parentales impliquées. Une consultation spécialisée en génétique est recommandée dès lors qu'une mutation est retrouvée. En collaboration avec le CRCM du LanguedocRoussillon, nous avons comparé la liste des patients présentant une ou deux mutations à la liste des patients vus en consultation de génétique. Nous avons reçu $38 \%$ des parents d'enfant avec une ou deux mutations. Parmi ces patients, nous avons vu une proportion plus importante de parents d'enfants non malades $(42 \%)$ que de parents d'enfants malades $(26 \%)$. Les parents d'enfants non malades viennent beaucoup plus rapidement en consultation : plus de $50 \%$ viennent dans les 5 mois suivant la naissance de l'enfant. Les parents d'enfants malades viennent plus tardivement, dans la deuxième année de vie de l'enfant. Ces différences peuvent en partie s'expliquer par la priorité de la prise en charge pour les enfants malades et au fait que le prélèvement sanguin des parents est fait par le CRCM à la demande du laboratoire de diagnostic moléculaire pour vérifier la contribution bi-parentale des mutations. Ainsi les parents d'enfants malades viennent rarement pour un conseil génétique immédiat pour la mucoviscidose, mais seulement lorsqu'il y a un nouveau désir d'enfant ou une grossesse en cours. Bien que le dépistage néonatal n'ait pas pour vocation de dépister les nouveaux-nés hétérozygotes, le taux d'hétérozygotes parmi les « faux positifs » est très élevé ( $55 \%$ contre $4 \%$ attendu). Parmi nos patients, nous avons ainsi découvert un couple à risque $(1 / 46$, ce qui était statistiquement attendu pour un tel taux d'hétérozygotes). La Haute Autorité de Santé (HAS) a fait en janvier 2009 un état des lieux du dépistage néonatal de la mucoviscidose. Elle mentionne que la fédération des CRCM élabore des recommandations pratiques sur l'information à transmettre aux patients dans le cadre du conseil génétique. L'étude des données recueillies ici et la connaissance du fonctionnement du CRCM et du service de génétique en Languedoc-Roussillon nous incite à modifier notre façon de travailler ensemble. Ainsi, au cours de la première année de vie de l'enfant, une consultation de génétique sera proposée systématiquement par le CRCM aux parents d'enfants malades le même jour qu'une consultation de suivi de leur enfant.

Mots-clés : conseil génétique, mucoviscidose, dépistage néonatal.

EP65. FIRST REPORT OF A GERMINAL AND SOMATIC MOSAICISM FOR A MUTATION OF THE RYANODINE RECEPTOR TYPE 2 GENE: IMPLICATION FOR GENETIC COUNSELLING AND PATIENT CARING

N. Roux-Buisson (1), I. Denjoy (2, 3), F. Extramania (2), A. Leenhardt (2), P. Guicheney $(3,4)$, J. Lunardi $(1,5)$

(1) Biochimie et Génétique Moléculaire, CHU Grenoble, Grenoble, France; (2) Centre de Référence des Maladies Ryhtmiques Héréditaires, Hôpital Lariboisière, Paris, France ; (3) Inserm U956, G H Pitié-Salpêtrière, Paris, France ; (4) UMR 956, UPMC, Paris, France ; (5) Inserm U836, Grenoble Institut des Neurosciences, Grenoble, France
Catecholaminergic polymorphic ventricular tachycardia (CPVT) is a severe hereditary arrhythmogenic disease, characterized by syncope or sudden death induced by emotional or physical stress. The mortality rate in untreated individuals is $50 \%$ by age 40 . Early diagnosis through clinical and genetic screening is very useful in order to prevent sudden death by beta blockers treatment and/or implantable cardioverter defibrillator (ICD). Mutations in the cardiac isoforms of the ryanodine receptor gene (RYR2) and of the calsequestrin gene (CASQ2), which are involved in the calcium homeostasis in cardiomyocytes, have been associated with CPVT. We reported the case of a 7-year-old girl referred for CPVT. She was treated by nadolol and ICD was implanted after recurrent syncopal episodes and positive exercise stress test under treatment. CPVT was strongly suspected into her youngest brother who suddenly died during a soccer game. Molecular analysis by sequencing RYR2 in the index case identified a heterozygous c.7202G $>A$; p.Arg2401His mutation. Genetic screening by dHPLC and DNA sequencing showed that the mutation was absent in the asymptomatic father's and the alive siblings while it suggested the presence of a somatic mosaic in the mother. Unfortunately, DNA from the deceased child was not available. An amplification refractory mutation system (ARMS)-PCR assay confirmed the presence of the mutant allele in the mother's DNA. We performed a semi-quantitative assay by High Resolution Melting and determined a low level of mosaicism ranging between 5 and $10 \%$ in mother's buccal, urinary and leukocytes cells. Haplotyping using intragenic SNPs showed that the proband and her alive siblings inherited different alleles from the mother. Based on the somatic maternal mosaicism and the existence of two children presenting with a CPVT phenotype, occurrence of a germline maternal mosaicism was therefore highly probable. To our knowledge, this is the first reported case of mosaicism for a mutation of RyR2. This report must help to alert physicians and genetic counsellors for the possible presence of a mosaicism in families with RYR 2 mutations, even if standard methods failed to clearly detect a mutation in the father or the mother of the proband. In such a situation, sporadic cases of CPVT are usually thought to be caused by occurrence of a de novo mutation in the proband. In order to rule out the presence of a germline mosaicism in one of the parent, genetic screening of the siblings of a sporadic proband should be systematically realized. If a mutation occurs at a very early stage in the embryo the mosaicism would be germinal and somatic. In that case and depending on the level of mosaicism in the heart tissue, the parent that would present with such a mosaicism would therefore be at risk for CPVT and a specific caring will have to be set up. This illustrates the potential benefits of extensive genetic analysis in these CPVT families.

Mots-clés : CPVT, RyR2, mosaicism.

- P656. REMPLISSAGE D'UN QUESTIONNAIRE EN PRÉVISION DE LA CONSULTATION D'ONCOGÉNÉTIQUE ET VARIABLES FAMILIALES ET SOCIO-DÉMOGRAPHIQUES : QUELLES ASSOCIATIONS ?

D. Molière (1), P. Laurent-Puig (1), B. Buecher (1), K. Lahlou-Laforet (2) (1) UF d'Oncogénétique, HEGP (AP-HP), Paris, France; (2) Service de Psychologie Clinique et Psychiatrie de Liaison, HEGP (AP-HP), Paris, France

Contact : diane.moliere@egp.aphp.fr

Introduction et objectif: En amont de la consultation d'oncogénétique digestive et gynécologique de l'HEGP, un questionnaire est adressé aux futurs consultants afin de leur permettre de rassembler à l'avance leurs antécédents familiaux sur trois générations au moins. Ce procédé facilite le tracé de l'arbre généalogique qui est une étape essentielle pour décider de l'indication à un test génétique lors de la première consultation. L'objectif de cette étude rétrospective est d'évaluer le remplissage de ce questionnaire et de rechercher des associations entre ce taux et certaines variables. Patients et méthode : Les sujets inclus dans cette étude sont les 267 proposants vus en consultation d'oncogénétique par la conseillère en génétique, préalablement à la consultation avec l'oncogénéticien, entre janvier 2007 et juillet 2009. Selon le remplissage du questionnaire, les sujets ont été classés initialement en deux groupes : le premier est composé des consultants l'ayant rempli et le second des consultants ne l'ayant pas rempli. Dans un second temps, le premier groupe a été subdivisé entre les sujets ayant rempli exhaustivement le questionnaire (informations concernant les apparentés atteints et indemnes) et ceux l'ayant rempli partiellement (informations concernant uniquement sur les apparentés atteints). Les variables étudiées sont: le sexe, l'âge, le statut de parent, l'atteinte personnelle (quel que soit le type de prédisposition), le délai disponible pour effectuer ce travail, l'ancienneté du diagnostic personnel et le nombre d'apparentés atteints. Résultats : Les consultants ayant rapporté leur questionnaire rempli sont au nombre de $209(78 \%)$. Aucune des variables 
étudiées ne s'est révélée être un facteur associé à l'acceptabilité par les consultants. Cependant, plus le consultant a d'apparentés atteints, plus il a tendance à effectuer ce travail préparatoire $(\mathrm{p}=0,0518)$. Au sein du premier groupe, le remplissage exhaustif (148 sujets sur 209, soit $71 \%$ ) ou partiel (61 sujets sur 209 , soit $19 \%$ ) est par contre significativement associé à deux variables. Plus leur diagnostic personnel est récent, plus les consultants ont tendance à remplir partiellement le questionnaire $(p=0,021)$. De même, plus les consultants ont d'apparentés atteints, plus ils ont tendance à remplir partiellement le questionnaire $(p=0,013)$. Discussion : L'envoi systématique d'un questionnaire sur l'histoire familiale en amont de la consultation d'oncogénétique est donc un procédé bien accepté par les proposants. Par ailleurs, la qualité du remplissage semble dépendre du temps qui s'est écoulé depuis le diagnostic et du nombre d'apparentés atteints. La focalisation sur les apparentés atteints est probablement dépendante de l'angoisse liée à la maladie, amenant éventuellement les consultants à surévaluer leur risque. L'information délivrée à ce sous-groupe de consultants devrait être davantage personnalisée au moment de la consultation afin que le risque subjectif concorde mieux avec le risque réel.

Mots-clés : première consultation d'oncogénétique, questionnaire, histoire familiale.

EP657. HNPCC SYNDROME : IMPACT OF GENETIC COUNSELING ON THE RELATED

E. Chipoulet (1, 2), D. Bonnet (2), J. Selves (2), C. Toulas (1), L. Gladieff (1), V. Feillel (1), R. Guimbaud $(1,2)$

(1) Service d'Oncogénétique, Institut Claudius Regaud, Toulouse, France : (2) Pôle digestif. CHU de Toulouse, France

Contact : guimbaud.rosine@claudiusregaud.fr

Le syndrome HNPCC, lié à une mutation germinale d'un gène MMR, expose à un haut risque de cancers du côlon et de l'endomètre. L'identification de la mutation familiale permet la réalisation de tests prédictifs chez les apparentés majeurs. Des mesures efficaces de dépistage peuvent alors être proposées aux apparentés porteurs. Il est donc nécessaire que l'information diffuse au sein de la famille. À ce jour, en France, cette information ne peut s'effectuer que par l'intermédiaire des « cas index * ayant directement consulté en oncogénétique ; cette tâche peut être perçue avec difficulté. Le but de notre travail a été d'évaluer l'impact et les caractéristiques de la démarche oncogénétique des cas index HNPCC, sur leurs apparentés, en terme de test prédictif. Quarante-trois familles, pour lesquelles une mutation constitutionnelle délétère de l'un des gènes MMR a été identifiée en consultation d'oncogénétique et dont le résultat a été rendu avant juin 2007, ont été étudiées (âge moyen des cas index : 53 ans : 4 décédés pendant la procédure). Pour chaque famille les apparentés ayant eu un test prédictif ont été recherchés et leurs caractéristiques ont été recueillies. Les données provenaient exclusivement des dossiers familiaux oncogénétiques. Parmi les 583 apparentés vivants et majeurs identifiés sur les arbres généalogiques $\left(\mathrm{n}=196\right.$ au $1^{\text {et }}$ degré et $\mathrm{n}=387$ au 2,3 ou $4^{\circ}$ degré) : $119(20 \%)$ sont venus en consultation d'oncogénétique $(2 / 3$ d'apparentés au $1^{\text {er }}$ degré, soit 2 par famille et $1 / 3$ d'apparentés ? $2^{\mathrm{c}}$ degré, soit 1 par famille). Cinquante cinq \% des apparentés au $1^{\text {et }}$ degré venus en consultation étaient des femmes contre $72 \%$ pour les apparentés ? $2^{\mathrm{c}}$ degré $(\mathrm{p}<0,05)$. Il n'y avait pas de modification du taux de participation des apparentés au $1^{\text {er }}$ degré, ni des apparentés ? $2^{\mathrm{C}}$ degré, en fonction du sexe du cas index (le taux de participation des apparentés au $1^{\text {er }}$ et ???.

Mots-clés : syndrome HNPCC, Conseil génétique, diagnostic prédictif.

IP658. «LES COULEURS DE JEANNE \#: UN LIVRE POUR EXPLIQUER LA POLYPOSE ADÉNOMATEUSE FAMILIALE AUX ENFANTS À RISQUE

S. Fosse (2, 3), S. Maddonni (4), Roche Diagnostics (5), C. Colas (1)

(1) Département de Génétique, Groupe Hospitalier Pitié-Salpêtrière, AP-HP, Paris, France; (2) Service de Pédopsychiatrie, Groupe Hospitalier Pitié-Salpêtrière, AP-HP, Paris, France ; (3) Service de Pédopsychiatrie, CHR, Laval, France; (4) Dessinatrice, France; (5) Société Roche Diagnostics, France

Contact : chrystelle.colas@psl.aphp.fr

La Polypose Adénomateuse Familiale (PAF) est une prédisposition au cancer colorectal de transmission autosomique dominante liée au gène APC. Les patients présentent de très nombreux polypes adénomateux au niveau colorectal dont l'évolution aboutit systématiquement au cancer en l'absence d'un diagnostic précoce et d'une prise en charge appropriée par une colectomie prophylactique. Les enfants des patients atteints ont $50 \%$ de risque d'avoir reçu la mutation du gène APC responsables de la polypose. Les polypes peuvent commencer à se développer dans l'enfance et les recommandations de prise en charge préconisent de débuter la surveillance endoscopique vers 12 ans. En France, le test génétique chez ces enfants mineurs est habituellement proposé à partir de 10 ans. Il est maintenant bien établi que les tests génétiques chez les mineurs doivent être encadrés et accompagné d'un conseil génétique approprié pour s'assurer de la compréhension de la maladie et du test génétique. Notre expérience avec ces enfants à risque de polypose venant pour le test génétique a mis en évidence que les discussions autour de la maladies étaient souvent difficiles voire inexistantes entre ces enfants et leurs parents. Les informations sur la transmission génétique et les risques de cette maladie sont des notions complexes qui sont difficiles à aborder avec de jeunes enfants. Pour améliorer la communication au sein de ces familles et aider les professionnels qui s'occupent du conseil génétique dans cette maladie, nous avons créé un livre à destination de ces enfants. Une dessinatrice professionnelle a illustré l'histoire de Jeanne, une jeune fille dont le père a une PAF et qui va elle-même faire le test génétique. Roche Diagnostics nous a aidé à concrétiser ce projet en nous apportant une aide technique et financière. Ce livre a été envoyé à tous les centres de Français d'oncogénétique qui travaillent sur cette maladie et est disponible sur demande. Nous espérons qu'il aidera à améliorer la prise en charge de ces enfants et de leur famille.

Mots-clés : polypose, test présymptomatique, mineurs. DP659. ÉVALUATION DE LA TRANSMISSION DE LINFORMA-
TION AUX APPARENTÉS DANS LES FAMILLES À RISQUE DE
PARAGANGLIOMES/PHEOCHROMOCYTOMES INTÉRETT DE
LA CGH ARRAY

C. Oheix $(1,2,3)$, T. Kogut-Kubiak $(1,2,3)$, P. Van Vaeck $(1,4)$, K. Lahlou-Laforêt $(1,4)$, A.P. Gimenez-Roqueplo $(1,2)$

(1) Assistance Publique-Hôpitaux de Paris, consultation multidiscplinaire d'oncogénétique, phéochromocytomes, paragangliomes et autres tumeurs endocrines, Hôpital européen Georges-Pompidou, Paris, France: $(2$ Assistance Publique-Hôpitaux de Paris, Hôpital Européen Georges-Pompidou, Service de génétique, Paris, France; (3) Étudiante de master 2 conseil en génétique-Université de la Méditerranée, Aix-Marseille II, France; (4) Assistance Publique-Hôpitaux de Paris, Hôpital Européen Georges-Pompidou, Service de Psychologie Clinique et Psychiatrie de Liaison, Paris, France

Contact : anne-paule.gimenez-roqueplo@egp.aphp.fr

Introduction L'identification d'une mutation prédisposant aux formes héréditaires de paragangliomes/phéochromocytomes présente, outre l'intérêt direct pour le sujet testé, un intérêt pour ses apparentés qui souhaiteraient connaître leur statut génétique, à condition qu'ils aient été informés. Nous disposons de peu de moyens standardisés pour apprécier la transmission de cette information par le sujet testé. Objectif de l'étude L'objectif de cette étude est d'évaluer la transmission effective de l'information génétique dans les familles mutées sur un gène de prédisposition, et de tester un outil permettant d'évaluer cette transmission. Patients et Méthodes Après avoir repris l'ensemble des dossiers des 890 consultants qui ont été reçus par la consultation multidisciplinaire d'oncogénétique des tumeurs endocrines de l'HEGP pour une suspicion de prédisposition héréditaire au phéochromocytome et/ou au paragangliome, nous avons selectionné les familles chez lesquelles une mutation sur SDHB, SDHD, SDHC, VHL ou RET a été identifiée. Nous avons estimé la transmission du risque génétique comme effective lorsque nous avions connaissance de l'ensemble des résultats de dépistage des apparentés à risque, ou éventuellement sur l'affirmation d'un membre de la famille que les apparentés non encore testés ont été correctement informés. La transmission a été estimée comme incertaine lorsque le dépistage familial ne semblait pas finalisé alors que la dernière consultation avec un membre de la famille remontait à plus d'un an. Un questionnaire comportant trois questions rédigé à cet effet a été adressé à la personne chargée de transmettre l'information auprès de ses apparentés, afin de savoir si ces derniers ont été informés et/ou s'ils ont réalisé le dépistage génétique. Une nouvelle consultation centrée sur les éventuels problèmes posés par la transmission de l'information est proposée à la fin du questionnaire. Résultats Parmi les 268 familles suivies. 99 d'entre elles ont une mutation délétère identifiée. Le dépistage familial est terminé pour 30 familles et est en cours pour 22 autres. Le questionnaire a été envoyé en septembre 2009 à 34 familles pour lesquelles la transmission a été jugée incertaine afin d'évaluer la transmission de l'information génétique et/ou de relancer le processus de dépistage. Treize familles suivies par d'autres centres n'ont pas fait l'objet de l'envoi du questionnaire. Discussion et Conclusion Cette étude a permis de quantifier la transmission et d'identifier les familles rencontrant des difficultés à transmettre l'information génétique. Nous aurons les résultats du retour des questionnaires pour les $5^{\text {es }}$ Assises de Génétique. L'envoi du questionnaire pourrait 
devenir systématique après un an sans nouvelles d'une famille à risque, si cette méthode s'avérait concluante.

Mots-clés : information familiale, paragangliome, phéochromocytome.

\section{口P66. ÉTUDE FRANÇAISE RÉTROSPECTIVE DE L'INFLUENCE DE LA GROSSESSE SUR LES HÉMANGIOBLAS- TOMES DANS LA MALADIE DE VON HIPPEL-LINDAU}

C. Abadie (1, 11), I. Coupier (1, 2, 11), S. Bringuier-Branchereau (3), G. Mercier (4), F. Séguret (4), N. Aghakhani (5, 11), P. David (5, 11), F. Parker (5), D. Le Gars (6, 11), G. Robert (7), P. Pencalet (8), J.P. Houtteville (9), P. Mercier $(10,11)$, S. Deveaux (11), S. Richard $(11,12)$ pour le Réseau National et le Centre de Références INCA « Maladie de VHL et prédispositions héréditaires au cancer du rein de l'adulte »

(1) Service de Génétique Médicale, Unité d'Oncogénétique, CHU Montpellier, France; (2) Service d'Oncogénétique, CRLCC Val d'Aurelle, Montpellier, France ; (3) Service d'Anesthésie Réanimation A, CHU Montpellier, France ; (4) Département d'Informatique Médicale, CHU Montpellier, France; (5) Service de Neurochirurgie, Hôpital de Bicêtre, Le Kremlin-Bicêtre, France; (6) Service de Neurochirurgie, Hôpital Nord, Amiens, France; (7) Service de Neurochirurgie, Fondation Ophtalmologique Rothschild, Paris, France ; (8) Service de Neurochirurgie, Hôpital Foch, Suresnes, France; (9) Service de Neurochirurgie, Centre Hospitalo-Universitaire, Caen, France ; (10) Service de Neurochirurgie, Centre Hospitalo-Universitaire, Caen, France: (11) Réseau National et Centre de Référence Cancers Rares INCa «Maladie de VHL et prédispositions héréditaires au cancer rénal de l'adulte » (PREDIR), Service d'Urologie, AP-HP, Hôpital de Bicêtre, Le Kremlin-Bicêtre, France ; (12) Laboratoire de Génétique Oncologique EPHE, CNRS FRE 2939, Faculté de Médecine Paris-Sud, Le Kremlin-Bicêtre et Institut de cancérologie Gustave Roussy, Villejuif, France

Contact : abadie_caro@yahoo.fr

La maladie de von Hippel-Lindau (VHL) [OMIM 193300] est une affection héréditaire de transmission autosomique dominante prédisposant à de multiples tumeurs richement vascularisées : hémangioblastomes du système nerveux central (SNC) et de la rétine, cancers du rein à cellules claires, kystes rénaux et pancréatiques, phéochromocytomes, tumeurs neuroendocrines du pancréas et tumeurs du sac endolymphatique. Cette affection est liée à des mutations constitutionnelles délétères du gène suppresseur de tumeur VHL localisé dans la région 3p25-26 qui joue un rôle majeur dans la réponse tissulaire à l'hypoxie et l'angiogenèse. Les hémangioblastomes du SNC et de la rétine, manifestations emblématiques de l'affection, surviennent respectivement chez environ $75 \%$ et $60 \%$ des patients atteints par la maladie de VHL. Jusqu'ici, seules quelques observations isolées et une étude sur une petite série de patients [1] se sont interrogées sur le rôle potentiellement délétère de la grossesse sur les hémangioblastomes qui expriment fortement des récepteurs aux oestrogènes et à la progestérone. Nous avons donc réalisé une étude française rétrospective comparative chez 269 patientes issues de 172 familles extraites de la base de données VHL nationale et réparties en deux groupes selon leur statut gestationnel. L'étude s'est intéressée spécifiquement à l'apparition de nouveaux hémangioblastomes, à l'accroissement de lésions déja connues et à la survenue d'éventuelles complications cliniques nécessitant une prise en charge thérapeutique. Dans ce but, les données disponibles des imageries de surveillance du SNC et de la rétine ont été collectées chez 176 femmes ayant eu au moins une grossesse et chez 93 femmes nulligestes. À notre connaissance, il s'agit de la seule étude analysant l'effet de la grossesse sur une série composée d'un effectif de patientes aussi important dans la maladie de VHL. Les résultats préliminaires montrent un nombre significativement plus élevé de nouvelles tumeurs et de complications dans le groupe des patientes ayant été enceintes. En particulier, l'exérèse chirurgicale d'hémangioblastomes du SNC a été nécessaire au cours de la grossesse chez trois patientes en raison d'une hypertension intracrânienne liée à une augmentation de la taille tumorale. Trois autres patientes ont subi une exérèse tumorale en urgence dans le premier mois du post-partum. En conclusion, l'augmentation potentielle de taille des hémangioblastomes du SNC ou l'apparition en quelques semaines d'un kyste compressif souligne la nécessité d'une surveillance étroite des patientes avec maladie de VHL au cours de la grossesse. Une IRM sans injection de Gadolinium est ainsi systématiquement préconisée au quatrième mois de grossesse chez toute patiente présentant une atteinte préalable du SNC.

\section{Référence}

1. Grimbert et al. Pregnancy in von Hippel-Lindau disease. Am J Gyn Obst 1999 ; $180: 110-1$

Mots-clés : maladie de von Hippel-Lindau, grossesse, hémangioblastomes.
IP661. 5 ANS DE RECUL SUR LA CONSULTATION PSYCHOLOGIQUE EN ONCOGÉNÉTIQUE À L'INSTITUT DE CANCÉROLOGIE GUSTAVE ROUSSY

I. Léger (1), V. Byrde (2), E. Consolino (2), O. Caron (2), S. Dauchy (1) (1) Unité de psycho-oncologie, DISSPO, IGR, Villejuif, France; (2) Consultation d'oncogénétique, département de médecine, IGR, Villejuif, France

Contact : isabelle.leger@igr.fr

La mise en place initiale de la prise en charge multidisciplinaire en oncogénétique à l'Institut Gustave Roussy, en 2004, reposait sur une proposition systématique de la consultation psychologique par le généticien, mais de façon disjointe de la sienne. La proportion de patients ultérieurement rencontrés par la psychologue restait faible $(3,5 \%)$. Or l'intervention du psychologue dans la démarche d'oncogénétique est souvent nécessaire. En effet, au-delà de la prévention, de l'évaluation et de la gestion du retentissement émotionnel, d'autres missions lui incombent : soutien du demandeur, évaluation des motivations et du contexte de la démarche, l'accompagnement à la transmission familiale de l'information, aide à la gestion de l'information et de l'incertitude. Autant d'objectifs souvent méconnus du patient, et qui ajoutés aux habituelles résistances rendent précaire le fait de faire reposer leur accès au psychologue sur leur simple demande en début de démarche. Nous avons donc depuis début 2007 choisi de proposer plutôt des consultations en binôme dans les situations les plus difficiles (Foster (2002), Hopwood (2005)), pour les patients de moins de 30 ans lors de la première consultation de génétique, et pour les rendus de résultats positifs ou non. Cette consultation en binôme systématique (sauf refus du patient) a permis d'augmenter la proportion de patients rencontrés par la psychologue à la suite de ce premier contact $(27 \%)$. Nous avons évalué la pertinence de cette augmentation en évaluant la perception de la présence du psychologue par les patients à l'aide d'un sondage (500 questionnaires distribués avec 400 réponses). Au moment de la première consultation de génétique avec la psychologue, $65 \%$ des patients ont souhaité sa présence au moment du rendu de résultat. À noter que $42 \%$ des patients ont également souhaité sa présence au moment du rendu de résultat alors qu'elle avait été absente lors de la première consultation de génétique. Concernant les rendus de résultats positifs, la présence de la psychologue a rassuré les patients dans $50 \%$ des cas, dans $45 \%$ cela n'a rien changé et aucun patient ne dit n'avoir été gêné. Lors des rendus de résultats négatifs, $44 \%$ de patients étaient rassurés par sa présence, pour $44 \%$ d'entre eux cela n'a rien changé et dans $9 \%$ des cas ils ont été gênés. En conclusion, notre organisation en binôme ne semble pas gêner les patients et permet de passer d'un taux de suivis psychologiques de $3,5 \%$ à $27 \%$.

Mots-clés : impact psychologique, oncogénétique, organisation.

EP662. QUEL EST LE NOMBRE DE SUJETS QUI NE VIENNENT FINALEMENT PAS EN CONSULTATION D'ONCOGÉNÉTIQUE ? UNE ESTIMATION MONOCENTRIQUE

E. Consolino, V. Byrde, D. Malka, O. Caron

Consultation d'Oncogénétique, Département de Médecine, Institut Gustave Roussy, Villejuif, France

Contact : olivier.caron@igr.fr

Contexte : Le nombre (nb) de sujets (sj) vus en consultation d'oncogénétique (CSO) croît de manière importante en France. En revanche, le nb de sj ne venant pas à une CSO prévue reste mal connu. Méthodes: Dans cette étude monocentrique à partir de données prospectives, nous avons comptabilisé sur l'année 2008 (date de point : 30/09/09) : 1) le nb de CSO programmées pour des sj adressés « en interne " par des médecins de notre centre (rendez-vous programmé dès lors que le sj donne son accord pour une $\mathrm{CSO}$ ) ; 2) le nb de contacts pour des sj « de l'extérieur » (demande d'avis par courrier ou téléphone de leur médecin,...), systématiquement répertoriés dans l'attente d'une prise de contact de leur part; 3) le nb de CSO effectivement réalisées (total et en fonction du mode d'adressage du patient, « interne » ou « externe ») ; le nb de CSO 4) prévues et 5) réalisées pour rendu de résultats à des sj apparentés asymptomatiques (APS). Résultats : Sur 1486 CSO de cas index prévues en 2008, 1370 (92\%) ont été réalisées à la date de point, soit un taux d'attrition de $8 \%$ (116 sj : 73 des 822 sj adressés « en interne » $[8,9 \%]$ et 43 des 664 sj adressés « de l'extérieur " $[6,5 \%])$. Sur 109 CSO prévues pour rendu de résultats à des APS, 13 n'ont pas été réalisées (12\%). Conclusion: Le taux d'attrition global de notre activité de CSO est de $8 \%$. Ce taux, relativement bas, est probablement surestimé dans la mesure où il ne tient pas compte des sj vus en CSO dans un autre centre ou qui viendront en CSO dans notre centre au-delà de la date de point. Bien qu'il semble a priori plus pratique aux sj adressés en interne de venir en CSO, le taux d'attrition de cette population $(8,9 \%)$ est supérieur à celui des $\mathrm{sj}$ « de l'extérieur » $(6,5 \%)$; cependant, ce dernier est probablement sous-estimé, ne tenant pas compte de sj 
pour lesquels le médecin extérieur n'a pris aucun contact. Au total, les contraintes d'adressage en CSO (impossibilité de convoquer un sj, démarche volontaire et active de celui-ci, accessibilité limitée des CSO) ne semblent pas avoir un impact fort sur le taux d'attrition de notre CSO, inférieur à celui observé pour rendu de résultats à des APS (12\%), pourtant a priori « motivés ». Ces données devraient être vérifiées par une étude prospective multicentrique, visant notamment à préciser les causes de non venue en CSO.

Mots-clés : oncogénétique, test génétique, taux d'attrition.

IP663. COMPLICATION DE LA CHIRURGIE GASTRIQUE PROPHYLACTIQUE POUR MUTATION DU GENE CDH1

J.P. Fricker, C. Degermann, H. Nehme-Schuster, P. Dufour

Unité d'oncogénétique, CLCC Paul-Strauss, Strasbourg, France

Contact : Jfricker@strasbourg.fnclcc.fr

Les mutations du gène $\mathrm{CDH} 1$ sont responsables d'une prédisposition au cancer gastrique diffus héréditaire, à laquelle s'ajoute chez les femmes un risque de cancer lobulaire du sein. La découverte d'une mutation du gène $\mathrm{CDH} 1$ chez un sujet asymptomatique conduit à recommander une gastrectomie prophylactique. Nous rapportons le cas d'une gastrectomie totale réalisée à titre prophylactique chez un homme de 46 ans porteur asymptomatique d'une mutation du gène $\mathrm{CDH} 1$. Les complications du geste chirurgical ont entrainé une morbidité prolongée, nécessitant un arrêt de travail plus de 6 mois après l'intervention, et une possible réintervention. Les indications de la gastrectomie prophylactique dans ce contexte sont peu nombreuses, et les complications difficiles à évaluer. Comme pour toute chirurgie prophylactique, il importe de prendre en compte le rapport bénéfice/risque, et les complications médicales, psychologiques et socio-professionnelles du geste proposé. En raison de ses implications médicolégales possibles, cette observation est l'occasion de rappeler la nécessité impérieuse d'une décision multidisciplinaire impliquant chirurgien, spécialiste d'organe, oncogénéticien et psychologue.

Mots-clés : prédisposition génétique, $\mathrm{CDH1}$, prophylaxie chirurgicale.

aP664. MIRAGE : GESTION DES DONNÉES CLINIQUES SOUS FILEMAKER PRO

A. Laugé (1), S. Miglierina (1), I. Eugène (1), C. Rougeron (1), C. Gilbon (1), M. Belotti (1), D. Stoppa-Lyonnet $(1,2)$

(1) Génétique oncologique, Institut Curie, Paris, France; (2) Université Paris Descartes, Paris, France

Contact : alg@curie.net

Actuellement l'ensemble des données cliniques du laboratoire de génétique de l'Institut Curie sont saisies dans un logiciel développé sous $4 \mathrm{D}^{\mathrm{TM}}$ : Gensource $^{\circledR}$. Ce logiciel a été développé par la société Micro6@ en partenariat avec le Pr D. Stoppa-Lyonnet entre 1992 et 1994 et montre aujourd'hui certaines limites de souplesse et d'évolutivité. Depuis fin 2004, la gestion des analyses, des résultats et des comptes rendus est faite grâce à une base de données sous FileMaker Pro ${ }^{\circ}$. Cette migration ayant été une réussite, nous avons retenu le même modèle pour gérer les données cliniques. De plus, ceci permettait des développements futurs plus faciles. Le développement de MIRAGE (Module Informatisé de Référencement des Analyses GEnétiques) a été confié à C. Baldassara (prestataire de service ayant réalisé la base dédié au laboratoire) en collaboration avec le référent SIH (Système d'Information Hospitalier) du service. Le cahier des charges de MIRAGE a été établi conjointement avec les secrétaires médicales en adéquation avec leur organisation de travail. MIRAGE s'articule autour d'un enregistrement du patient (vu ou non en consultation) et de plusieurs enregistrements satellites : renseignements sur la famille, tumeurs développées, prélèvements disponibles, études génétiques, consultations, courriers, participation à différentes études. À chaque patient est attribué un identifiant unique composé d'un numéro de famille et d'un numéro d'individu dans la famille. Ce numéro permet de regrouper facilement les patients par famille et de retrouver les informations relatives à leur famille. En plus d'avoir une structure simple pour la maintenance et la navigation, les principaux avantages de MIRAGE sont de (i) centraliser les informations disponibles au sein du service pour chaque patient, (ii) simplifier les saisies pendant les consultations, (iii) récupérer automatiquement les informations du patient grâce à son numéro personnel d'identification. (iv) automatiser l'impression des ordonnances ou l'assignation dans une série d'analyse, (v) visualiser les données du patient disponibles dans l'Hôpital, (vi) générer un courrier type et (vii) exporter les données de la famille pour le dessin de l'arbre familial et le calcul de risque. Le dossier patient informatisé permettra une disponibilité immédiate des informations du patient et donc un gain de temps pour les membres du service. De plus la centralisation des informations permet de croiser les données cliniques et les données d'analyses afin de mettre en évidence des corrélations génotype/phénotype ou de créer des cohortes d'étude par simple requête. MIRAGE permet un élargissement des champs d'application en offrant la possibilité d'approfondir les connexions avec les autres bases de l'hôpital et d'inclure les documents numérisés relatifs aux patients.

Mots-clés : Filemaker Pro, base de données, génétique.

E P665. L'OFFRE DE TESTS GÉNÉTIQUES EN FRANCE : COMMENT OPTIMISER LE DISPOSITIF ?

M. Jovanovic, I. Caron, A. Rath, M. Hanauer, S. Aymé

Inserm, SCII - Orphanet, Paris, France

Contact : segolene.ayme@inserm.fr

La base de données Orphanet s'efforce depuis douze ans de collecter l'information sur les tests disponibles pour le diagnostic des maladies rares, en France et dans une trentaine de pays européens, en particulier grâce à un partenariat avec EuroGentest. Toutes les maladies mendéliennes étant rares, cela inclut donc une collecte de l'information sur les tests génétiques. Elle est effectuée auprès des laboratoires publics et privés avec une mise à jour annuelle. Elle repose sur les déclarations des professionnels responsables. En Septembre 2009, le nombre de laboratoires effectuant des tests de génétique moléculaire en France est de 181. Ces laboratoires testent 861 gènes sur les 1467 gènes testés en Europe. Aucun pays européen n'est en mesure d'offrir tous les tests, ce qui montre la nécessité d'organiser les règles de coopération entre pays européens. Seules les mutations du gène CFTR sont analysées dans tous les pays. Les tests les plus disponibles par ordre décroissant sont: mucoviscidose, hémochromatose, myopathie de Duchenne, $\mathrm{X}$ fragile, surdité non syndromique, Angelman, cancer du sein familial, deletion de l'Y, Huntington, Prader-Willi, cancer du colon non polyposique familial, déficit en facteur II et facteur V, syndrome de Rett, syndrome de Williams, myotonie de Steinert et Achondroplasie. Ces données interrogent sur l'organisation optimale de l'offre de tests au moment où la France régionalise son système de santé et regroupe ses laboratoires dans de grandes plateformes. À l'évidence les tests génétiques ne doivent pas être vus comme devant faire partie de l'offre régionale, mais à intégrer dans une politique nationale et européenne. Les données région par région seront présentées et des solutions possibles à une organisation de l'offre seront discutées.

Mots-clés : tests génétiques, organisation des soins, génétique moléculaire.

EP66. ÉTUDE DE LA PERCEPTION DES PATIENTS FACE À LA RÉCEPTION D'UNE COPIE DU COMPTE RENDU ADRESSÉ À LEURS MEDECINS RÉFÉRENTS À L'ISSUE DE LA CONSULTATION DE GÉNÉTIQUE

C. Cassini (1), C. Thauvin-Robinet (1), S. Vinault (2), C. Binquet (2), F. Coron (1), S. Mercier (3), N. Laguette (3), N. Herman (3), L. Faivre (1) (1) Centre de Génétique, Hôpital d'enfants, CHU Bocage Dijon; (2) Centre d'Investigations Clinique - Épidémiologie Clinique, Faculté de Médecine, CHU Dijon : (3) Direction des Droits des Patients, de la Qualité et de la Gestion des Risques, CHU Bocage, Dijon

Contact : cecile.cassini@chu-dijon.fr

Les recommandations de bonnes pratiques de l'HAS pour l'information des patients, éditées en Mars 2000, rappellent l'obligation d'information du patient par le corps médical sur son état de santé. La primauté de l'information orale est pointée, les documents écrits pouvant en être un complément. Ces données sont reprises dans la loi du 4 Mars 2002 relative au droit des patients. Il apparaît que les pratiques des différents services de Génétique en France sont très hétérogènes (envoi systématique, à la demande, courrier spécifique aux patients...). Avant de généraliser l'envoi du double du courrier médical au décours de leur consultation de génétique, nous avons souhaité initier, dans le cadre d'une évaluation des pratiques professionnelles, une étude visant à évaluer l'intérêt, le ressenti et surtout l'absence de caractère délétère de la réception de ce courrier par les parents/ patients. Au cours de 4 mois d'activité de 2 médecins généticiens et d'une conseillère en génétique du centre de Génétique du CHU de Dijon ( $1^{\text {er }}$ septembre-31 décembre 2008), il a été proposé aux patients de recevoir un double du courrier adressé aux médecins référents. Sur un total de 379 consultations, 353 questionnaires ont été envoyés. 15 patients (4\%) ont refusé de recevoir ce double du courrier, et le consultant a jugé cet envoi inadapté pour 11 patients (3\%, en général quand un enfant n'était pas accompagné de ses parents). 226 questionnaires ont été retournés $(60 \%)$. Les consultations concernaient des adultes dans $73 \%$ des cas, des enfants dans $27 \% .32 \%$ des consultations avaient un but diagnostique, $47 \%$ de conseil génétique, $14 \%$ de résultats et $7 \%$ de suivi. $99 \%$ des patients ont été satisfait de recevoir ce double du courrier. Ce courrier a été perçu comme une aide à la compréhension des informations médicales 
dans $72 \%$ des cas (et ce d'autant moins que le niveau d'études est élevé), et cela a permis de retrouver des informations oubliées après la consultation pour $17 \%$ des patients. Pour $82 \%$ des patients, ce courrier était considéré comme un reflet fidèle de la consultation, pour les $18 \%$ restants, le courrier était plutôt jugé plus complet que la consultation. Les termes employés ont été ressentis comme compréhensibles dans $97 \%$ des cas, mais trop médicaux dans $20 \%$, et choquants dans seulement $3 \%$ des cas. II n'existait pas de différence pour les différents paramètres suivant le motif de la consultation, ou si le patient était un enfant ou un adulte. $65 \%$ des patients faisaient part de leur intention d'utiliser par la suite cette copie, pour la communication avec le milieu médical et/ou la famille, ou pour initier des démarches particulières. $63 \%$ signalent habituellement récupérer secondairement une copie du courrier envoyé par leur médecin référent. Enfin, $58 \%$ des patients souhaiteraient plutôt un courrier spécifique adapté, qui leur serait personnellement destiné. En conclusion, cette étude montre la satisfaction des patients face à cette démarche, et l'absence de caractère délétère de celle-ci. La réception de ce courrier assure une meilleure intégration des données transmises lors de la consultation par le patient, et représente un support écrit qui peut faciliter la transmission correcte des informations à la famille.

Mots-clés : information médicale, questionnaire patients, évaluation des pratiques professionnelles.

\section{EP667. CODIFICATION ET CLASSIFICATION DES MALADIES GÉNÉTIQUES POUR LES BESOINS DES SYSTÈMES DE SANTÉ ET CEUX DE LA RECHERCHE SCIENTIFIQUE}

A. Rath, B. Bellet, Marie Georget, S. Aymé

Inserm, SC11 - Orphanet, Paris, France

Contact : segolene.ayme@inserm.fr

Les systèmes d'information sont maintenant des outils indispensables à la gestion des systèmes de santé comme à la recherche biomédicale. Ils nécessitent la standardisation des concepts à l'intérieur d'un groupe d'usage. Ainsi le codage des maladies se doit de refléter la réalité perçue par les professionnels de santé, par les patients et leur entourage, les noms de maladie servant à communiquer. $\mathrm{Ce}$ codage doit pouvoir servir aussi la Santé Publique, en organisant les maladies hiérarchiquement, permettant ainsi des agrégations à des fins statistiques, organisationnelle ou économiques. C'est le codage de la morbidité et de la mortalité. Les chercheurs travaillant sur l'étiologie, la physiopathologie et les cibles thérapeutiques ont d'autres besoins et d'autres types de représentation. Ils ont besoin de manipuler l'information au niveau des phénomes avec un niveau de granularité le plus fin possible (corrélation génotype-phénotype). Le degré de granularité de l'information nécessaire peut ainsi varier grandement. La Classification Internationale des Maladies (CIM10) est adaptée aux besoin des gestionnaire de la santé mais insatisfaisante pour les besoins des professionnels de santé en génétique car seules 200 maladies génétiques ont un code CIM spécifique. Il en va de même de Snomed CT. Le catalogue OMIM est largement utilisé par les généticiens et les chercheurs mais il n'est pas adapté au codage des bases de données de patients car c'est un catalogue de gènes et de phénotype comme proxy de gène, et non un catalogue de maladies au sens clinique du terme. Dans ce contexte, Orphanet a développé un système de codage et une classification polyhiérarchique maintenant à la disposition de la communauté internationale. Chaque niveau de granularité est interfacé avec les autres systèmes de codage, permettant un transcodage automatique. Les choix conceptuels seront explicités et les modes d'utilisation de la nomenclature Orpha seront présentés. Celle-ci sert actuellement à la révision de la Classification Internationale des Maladies (vers la CIM11), processus ouvert auquel tous les généticiens devraient s'intéresser en raison des enjeux pour la visibilité des maladies génétiques dans les systèmes de santé.

Mots-clés : codage des maladies génétiques, classification des maladies génétiques, systèmes d'information.

\section{P668. ORPHANET VU PAS SES UTILISATEURS : LEÇONS} POUR L'AVENIR

C. Rodwell, C. Angin, M. Hanauer, S. Aymé

Inserm, SCII - Orphanet, Paris, France

Contact : segolene.ayme@inserm.fr

Orphanet est un portail d'information sur les maladies rares, dont toutes les maladies génétiques. Créé il y a douze ans, il en est à sa quatrième version majeure pouroffrir des services répondant aux besoins de ses utilisateurs. Le nouveau portail fut lancé en Avril 2008, suivi un après d'une enquête. Le questionnaire était proposé en ligne via un un pop up sur les différentes pages. L'internaute avait le loisir de répondre à l'enquête ou d'ignorer l'invitation. La durée de cette enquête fut limité à l'obtention de 1000 questionnaires en langue française remplis, conformément à ce qui avait été fait par le passé. Les utilisateurs sont pour $61 \%$ des professionnels, pour $26 \%$ des malades, familles ou entourage et pour $13 \%$ des personnes autres. Parmi les professionnels, ce sont les médecins hospitaliers qui sont les plus nombreux ( $23 \%$ de tous les professionnels), les médecins généralistes en représentant $9 \%$. La ventilation détaillée est très semblable à celle des enquêtes précédentes. Les utilisateurs francophones viennent de France $(80,5 \%)$, du Canada ( $5 \%$ ), d'Algérie $(2,7 \%)$, de Suisse $(2,2 \%)$, de Belgique $(2,1 \%)$, de Tunisie $(1,9 \%)$, du Maroc $(1,7 \%)$ et de plus de cinquante autre pays. Plus de $46 \%$ des utilisateurs sont titulaires d'un doctorat et seulement $3,4 \%$ n'ont aucun diplôme, montrant une fois de plus la fracture numérique. Les femmes représentent les deux tiers des internautes du site. Google est le mode majoritaire de découverte du site $(46 \%)$, suivi par le conseil d'un médecin $(14 \%)$, un site d'hôpital $(8 \%)$, puis un site d'association de malades $(7 \%)$. L'information la plus recherchée est l'information sur une maladie précise $(85 \%)$, puis celle sur un laboratoire de diagnostic $(20 \%)$, sur une consultation experte $(18 \%)$ et sur une association de malades $(17 \%)$. Orphanet a des utilisateurs réguliers : $27 \%$ viennent plus de deux fois par an, ! $32 \%$ plus de deux fois par mois et $19 \%$ plus de deux fois par semaine. Les quatre produits les plus plébiscités sont les textes sur les maladies, la nomenclature et les classifications, puis la recherche par signe, enfin les recommandations pour la pratique d'urgence, les listes de centres de référence et le répertoire des laboratoires médicaux. Les utilisateurs d'Orphanet sont aussi utilisateurs de PubMed $(43 \%)$, de Wikipedia $(37 \%)$, de sites de sociétés savantes $(18 \%)$ et d'OMIM $(17 \%)$. Les suggestions d'amélioration portent essentiellement sur une demande d'actualisation du site plus fréquente, sur une information plus détaillée sur chaque maladie, sur plus de convivialité dans la recherche par signe et sur l'ajout de banques d'image, mais surtout, surtout... sur la possibilité de faire des demandes à des experts pour des cas cliniques non résolus. Comment aller vers cette téléexpertise?

Mots-clés : base de données, Internet, Information.

\section{E669. CARACTÉRISTIQUES DES PATIENTS ATTEINTS} D'HTAP PORTEURS D'UNE MUTATION ACVRL1

B. Girerd (1, 2), D. Montani (1), F. Coulet (3), B. Sztrymf (1), A. Yaici (1), X. Jaïs (1), D. Tregouet (3), O. Sitbon (1), G. Simonneau (1), F. Soubrier (3), M. Humbert (1)

(I) Centre National de Référence de l'Hypertension Pulmonaire Sévère, Service de Pneumologie et Réanimation Respiratoire, Hôpital AntoineBéclère, AP-HP, Clamart, France ; (2) AFCG, Association Française des Conseillers en Génétique, Marseille, France; (3) Université Pierre-etMarie-Curie-Paris 6, Laboratoire d'Oncogénétique et Angiogénétique Moléculaire, UMRS 956 INSERM, Groupe Hospitalier Pitié-Salpétrière, Paris, France

\section{Contact : barbara.girerd19@gmail.com}

Introduction: Les mutations du gène ACVRL1 prédisposent pour la maladie de Rendu-Osler et/ou pour l'hypertension arterielle pulmonaire (HTAP). L'objectif de cette étude est de décrire les caractéristiques des patients ayant une HTAP et une mutation du gène ACVRL1. Méthodes : Les caractéristiques cliniques, fonctionnelles et hémodynamiques de 32 patients ayant une HTAP et une mutation ACVRL1 ont été analysées (9 patients suivis dans le centre national de référence de l'HTAP). Les caractéristiques de ces patients ont été comparées à celles de 370 patients souffrant d'HTAP (93 ayant une mutation BMPR2, et 277 n'ayant pas de mutation identifiée). La distribution des mutations sur le gène ACVRL1 retrouvées chez les patients ayant une HTAP a été comparée à celle des patients avec un Rendu-Osler sans HTAP. Résultats : Au moment du diagnostic d'HTAP, les porteurs d'une mutation ACVRL1 sont significativement plus jeunes $(21,8+/-16,7$ ans) que les porteurs d'une mutation BMPR2 et que les non porteurs de mutation $(35,7+/-14,9$ et $47,6+1-16,3$ ans respectivement, $p<0,0001)$. Les porteurs de mutation ACVRL1 étaient caractérisés par une hémodynamique moins sévère au diagnostic, une absence de réactivité en aigu au NO et un pronostic plus sombre. Chez les patients ayant une HTAP, il a été retrouvé significativement plus de mutation du gène ACVRL1 dans l'exon 10 par rapport aux patients ayant un Rendu-Osler sans HTAP $(33,3 \%$ et $5 \%, p<0,0001)$. Conclusion: Les patients atteints d'HTAP et porteurs d'une mutation ACVRL1 sont caractérisés par un plus jeune âge au diagnostic, une hémodynamique moins sévère et un pronostic plus sombre que les autres patients ayant une HTAP, suggérant une progression plus rapide de la maladie.

Mots-clés : bone morphogenetic protein receptor type 2, BMPR2, hypertension artérielle pulmonaire, maladie de Rendu-Osler. 
EP670. CLINICAL PHENOTYPES OF COLAA1 MUTATIONS CARRIERS ARE EXTREMELY VARIABLE. IMPORTANCE FOR CLINICAL CARE AND GENETIC COUNSELING

M. Mine (1, 2), M.L. Moutard (3), D. Hervé (4), A. Roubertie (5), M. Sevin (6), D. Rodriguez (3), K. Maincent (3), E. Berger (7), F. Rouannet (8), B. Echenne (5), K. Vahedi (4), E. Tournier-Lasserve (1, 2, 9)

(1) Laboratoire de Génétique, Hôpital lariboisière, Paris, France ; (2) Inserm UMR-S 740, Paris, France ; (3) Service de neuropédiatrie, Hôpital Trousseau, Paris, France : (4) Service de neurologie, Hôpital lariboisière, Paris, France; (5) Service de neuropédiatrie, Hôpital Gui de Chauliac, Montpellier, France ; (6) Service de neurologie, CHU de Nantes, France, (7) Service de neurologie, CHU de Besançon, France; (8) Service de neurologie, CHU de Bordeaux, France; (9) UFR de médecine Paris7 Denis Diderot, Paris, France

Contact : tournier-lasserve@univ-paris-diderot.fr

Background: Col4Al encodes the alphal chain of type IV collagen, a major component of basement membranes, including vascular basement membrane. Mutations of Col4A lhave been shown in 2005 to cause porencephaly and Intra Cerebral Hemorrhage (ICH) in mouse and man. Only $14 \mathrm{Col} 4 \mathrm{~A} 1$ mutated families have been reported so far ; a variety of additional phenotypes including retinal arteriolar tortuosities, HANAC and Axenfeld Rieger syndromes have been detected in some of them. Intracerebral hemorrhage $(\mathrm{ICH})$ is the most severe manifestation in COL4A1 mutants. Prevention of ICH and genetic counseling are very important in carriers. One major question is when should we suspect a Col4Al mutation and conduct a genetic testing in both infants and adults showing an $\mathrm{ICH}$ (which occurs in $10 \%$ of stroke patients). Aims : our main objective is to establish the full spectrum of COL4A1 related syndromes Patients and families: 16 unrelated index patients and their relatives were included. The 52 COL4A1 exons and exon/intron boundaries were sequenced Results: 16 probands/ 8 affected relatives included. Age at clinical onset in probands ranged from pre/perinatal period or infancy ( 7 cases), childhood ( 3 cases), adult life ( 6 cases/17-60 yo). Early onset cases showed a combination of IUGR, developmental delay, microcephaly, seizures, ICH and leukoencephalopathy (all patients), porencephaly ( 3 patients), microphtalmia, congenital cataract and /or optic atrophy ( 3 patients). None of them showed detectable renal anomalies and retinal arteriolar tortuosities. $4 / 7$ of these patients have a de novo mutation and 3 of them have inherited their mutation from an asymptomatic parent ( 2 patients) or a parent showing only mild symptoms (glaucoma at 37 yo). In $4 / 6$ patients with an adult onset, the initial manifestation was an ICH associated with a leukoencephalopathy and no associated symptom. In the 2 last ones, diagnosis was supected on the association of leukoencephalopathy and microbleeds on an MRI performed for an unrelated reason. Mutations were a Gly substitution in COL4A1 triple helix $(9 / 16)$, a splice site mutation $(2 / 16)$, a del or dup mutation (2) and a Gly substitution in the $\mathrm{NCl}$ c-term domain (1). Conclusions: Col4A1 mutations may lead to very severe early onset manifestations and developmental delay caused by both de novo and inherited mutations. ICH and leukoencephalopathy, a commonly encountered association in adult stroke patients, can be the only manifestation in an adult patient. These data raise important issues for genetic counseling.

Mots-clés : COL4A1, hémorragie cérébrale, leucoencephalopathie.

EP71. MALADIE DE FABRY: ATTEINTE CÉRÉBRO-VASCULAIRE ET CARDIAQUE DES PATIENTS HÉMIZYGOTES EN INSUFFISANCE RÉNALE TERMINALE

T.C. Tran (1, 2), F. Colas (3), K. Benistan (1, 2), R. Carlier (3), F. Jabbour (4), C. Perronne (5), F. Bellazreg (5), D. P. Germain $(1,2,6)$

(1) Centre de référence de la maladie de Fabry et des maladies héréditaires du tissu conjonctif, CHU Raymond-Poincaré, Garches, France ; (2) Unité Fonctionnelle de Génétique Médicale, CHU Raymond-Poincaré, Garches, France; (3) Service de Radiologie, CHU Raymond-Poincaré, Garches, France; (4) Laboratoire de biochimie et génétique médicale, CHU Raymond-Poincaré, Garches, France : (5) Département de médecine aigue, CHU Raymond-Poincaré, Garches, France ; (6) Université de Versailles - St-Quentin-en-Yvelines, Versailles, France

Contact : dominique.germain@rpc.aphp.fr

Introduction : La maladie de Fabry (MF, OMIM \#301500) est une maladie héréditaire du métabolisme, de transmission génétique liée au chromosome $\mathrm{X}$, due à un déficit en alpha-galactosidase $\mathrm{A}$ conduisant à l'accumulation lysosomale de glycosphingolipides dans de nombreux types cellulaires. Le déficit enzymatique s'accompagne d'une maladie multisystémique avec une morbi-mortalité élevée essentiellement due à une insuffisance rénale chronique (IRC) aboutissant à la dialyse ou la transplantation, et des atteintes cardiaques et cérébro-vasculaires à type de cardiomyopathie hypertrophie (CMH) et d'accidents vasculaires cérébraux. Peu de données sont disponibles dans la littérature sur les relations entre ces différentes atteintes. Dans cette étude, l'atteinte cérébro-vasculaire a été évaluée pour le sous-groupe des patients en insuffisance rénale terminale bénéficiant d'une épuration extra-rénale : dialyse ou transplantation. Patients et méthodes : 14 patients hémizygotes (âge : $48 \pm 8$ ans, intervalle : $38-66$ ans) furent inclus dans cette étude. Le diagnostic fut dans tous les cas confirmé par une activité alpha-galactosidase A effondrée et l'identification de la mutation dans le gène GLA. Une IRM cérébrale avec injection et une échographie cardiaque furent réalisées chez tous les patients pour étude de la vascularisation cérébrale et de la morphologie cardiaque, respectivement. Résultats : une ou des lacunes cérébrales furent identifiés $56 \%$ des cas (5/9), des séquelles d'accident vasculaire cérébral (AVC) dans $14 \%(2 / 14)$, une leucoencéphalopathie sévère avec un score de Fazekas entre 8 et 9 dans $50 \%$ des cas $(7 / 14)$, un diamètre élevé du tronc basilaire $(>4,7 \mathrm{~mm})$ dans $75 \%$ des cas mesurés $(9 / 12)$ et un diamètre élevé des artères cérébrales moyennes (MI $>3,7 \mathrm{~mm}$ ) dans $50 \%$ des cas (7/14). Un hypersignal T1 des pulvinars, signe très spécifique de la MF fut retrouvé dans $57 \%$ des cas. Enfin, 11 patients sur $14(79 \%)$ étaient porteurs d'une $\mathrm{CMH}$. Douze mutations différentes du gène GLA furent identifiées: p.Arg227Stop, p.Met42Arg, p.Cys52Arg, p.Phe295Cys, p.Gly183Asp, p.Gln306Stop, p.Lys168Asn, p.Ser345del2pb, p.Leu243Phe, p.Asp266Glu, p.Glu358Gly, p.Cys202Trp ; parmi lesquelles 2 mutations non-sens, 9 mutations faux-sens, et une délétion de petite taille. La majorité des mutations identifiées perturbe la structure tridimensionnelle de l'alpha-galactosidase A [2]. Aucune corrélation génotype - phénotype n'a pu être établie. Discussion : Une prévalence élevée d'AVC, de lacunes cérébrales, de leucoencéphalopathie sévère, de dolichoectasies et de $\mathrm{CMH}$ fut observée chez ces patients en IRC terminale, justifiant l'indication d'une thérapie enzymatique substitutive en prévention ou traitement des atteintes cardiaques et cérébrovasculaires qui ne sont pas corrigées par l'épuration extra-rénale.

\section{Références}

1. Germain DP. Fabry disease. Orphanet J Rare Dis 2009, sous presse.

2. Garman SC. Structure-function relationship in a-galactosidase A. Acta Paed. $2007: 96: 6-16$

Mots-clés : maladie de Fabry, IRM cérébrale, alpha-galactosidase.

a 672. LE PSEUDOXANTHOME ÉLASTIQUE: UNE CAUSE GÉNÉTIQUE DE DÉFICIT VISUEL SÉVÊRE CHEZ L'ADULTE JEUNE

K. Bénistan (1, 2), S. Mokhtari (3), C. Orssaud (4), R. Carlier (3), C. Vallée (3), X. Jeunemaitre (5), A.L. Fauret (5), C. Perronne (6), D.P. Germain (1, $2,7)$

(1) Centre de référence de la maladie de Fabry et des maladies héréditaires du tissu conjonctif, Hôpital Raymond-Poincaré, Garches, France ; (2) Service de Génétique médicale, Hôpital Raymond-Poincaré, Garches, France; (3) Service de Radiologie, Hôpital Raymond-Poincaré, Garches, France; (4) Service d'Ophtalmologie, Hôpital Européen Georges-Pompidou, Paris, France: (5) Service de Génétique, Hôpital Européen Georges-Pompidou, Paris, France ; (6) Département de Médecine Aiguë, Hôpital Raymond-Poincaré, Garches, France ; (7) Université de Versailles - St Quentin en Yvelines (UVSQ), France

Contact : karelle.benistan@rpc.aphp.fr

Le pseudoxanthome élastique (PXE, OMIM \#264800) est une maladie héréditaire du tissu conjonctif de transmission autosomique récessive, liée à des mutations du gène $\mathrm{ABCC} 6$ au locus 16p13. Les manifestations cliniques sont dermatologiques, ophtalmologiques et cardio-vasculaires, dues à des lésions calcifiantes des fibres élastiques. Les lésions vasculaires touchent les artères des membres, les coronaires, les axes carotidiens et vertébraux $[1,2]$. Nous rapportons l'observation d'une femme de 53 ans, née de parents non consanguins, chez laquelle un diagnostic de pseudoxanthome élastique fut posé en 2006 devant des lésions dermatologiques. Elle a présenté une atteinte ophtalmologique s'étant manifestée par une vision floue à 49 ans, en rapport avec des néovaisseaux maculaires. Son acuité visuelle était de $1 / 10$. Parinaud 20 à droite avec 2 lésions néovasculaires fibrosées au fond d'œil et de 6/10 Parinaud 2 faible à gauche avec des stries angiö̈des et une lésion néovasculaire choroïdienne en position rétrofovéale. Elle a bénéficié d'un traitement par photothérapie dynamique puis injections intra-vitréennes bilatérales de ranibizumab, ayant permis d'apparemment stabiliser les lésions. Sur le plan vasculaire, elle présentait des lésions d'artériosclérose précoce diffuses avec une artériopathie oblitérante des membres inférieurs (nécessité d'un pontage fémoro-poplité à 49 ans). Elle a par ailleurs présenté un déficit neurologique avec des troubles sensitifs de l'hémicorps gauche durant quelques semaines à l'âge de 51 ans, ayant révélé à l'IRM cérébrale de multiples hypersignaux punctiformes de 
la substance blanche bilatérale, une lacune cérébelleuse droite et un aspect grêle de l'artère carotide interne. La patiente est hétérozygote composite pour 2 mutations (c.4209 A(-2)>C) sur le site accepteur d'épissage de l'intron 29 et p.Trp1447Stop dans l'exon 30 du gène ABCC6. Cette observation illustre l'atteinte poly-viscérale communément retrouvée chez les patients atteints de PXE et la particulière gravité de l'atteinte ophtalmologique. Nous recommandons la réalisation systématique d'un fond d'œil tous les 6 à 12 mois et d'une angiographie rétinienne en cas de signe d'appel, afin de dépister précocement d'éventuelles complications. L'intérêt des nouvelles possibilités thérapeutiques ophtalmologiques (3), actuellement non enregistrées pour le PXE, devraient faire l'objet d'études contrôlées.

\section{Références}

1. Vanakker OM et al. Novel clinico-molecular insights in pseudoxanthoma elasticum provide an efficient molecular screening method and a comprehensive diagnostic flowchart. Hum Mutat 2008; $29: 205$.

2. Germain DP et al. Homozygosity for the R1268Q mutation in MRP6, the pseudoxanthoma elasticum gene, is not disease-causing. Biochem Biophys Res Commun 2000 Aug $2 ; 274$ (2): 297-301

3. Teixera A et al. Choroidal neovascularization treated with intravitreal injection of bevacizumab (Avastin) in angioid streaks. Acta Ophthalmol Scand 2006 ; $84(6)$ : $835-6$.

Mots-clés : pseudoxanthome élastique, ABCC6.

E673. LA DOULEUR DANS LE SYNDROME D'EHLERSDANLOS HYPERMOBILE

V. Martinez (1, 2), K. Benistan (3, 4), M.N. Gaveau-Glantin (5), D. Bouhassira (2), F. Roffi (6), C. Perronne (7, 8), D. Fletcher (1, 2, 8), D.P. Germain $(3,4,8)$

(1) Service d'Anesthésie Réanimation Chirurgicale, CHU Raymond-Poincaré, Garches, France; (2) INSERM, U-792, Centre d'Évaluation et de Traitement de la Douleur, CHU Ambroise Paré, Boulogne-Billancourt, France: (3) Centre de référence de la maladie de Fabry et des maladies héréditaires du tissu conjonctif (Mucopolysaccharidoses, pseudoxanthome élastique et syndromes d'Ehlers-Danlos), CHU Raymond-Poincaré, Garches, France; (4) Service de Génétique Médicale, CHU Raymond-Poincaré, Garches, France; (5) Association Française des Syndromes d'Ehlers-Danlos (AFSED), Tours, France; (6) Service de radiologie, $\mathrm{CHU}$ Raymond-Poincaré, Garches, France; (7) Département de Médecine Aigue Spécialisée, CHU Raymond-Poincaré, Garches, France ; (8) Université de Versailles - St Quentin en Yvelines, Versailles, France Contact : dominique.germain@rpc.aphp.fr

Introduction: Les syndromes d'Ehlers-Danlos (SED) sont un groupe de maladies héréditaires du tissu conjonctif. Le type hypermobile (OMIM $\# 130020$ ) est le plus fréquent rendant compte d'environ $60 \%$ des observations dans notre expérience. Il associe hyperlaxité articulaire, hyperelasticité cutanée, douleurs diffuses et asthénie. Alors que la douleur est un signe cardinal du SED hypermobile, son étiologie est mal connue et la littérature quasi-inexistante sur le sujet. Méthodologie : Étude prospective descriptive de la douleur chez des patients atteints du SED hypermobile adressés par le centre de référence des maladies héréditaires du tissu conjonctif. Recueil systématisé des caractéristiques de la population, du nombre et du type d'évènements douloureux, de la topopographie, de la durée et de l'évolution de la douleur. Évaluation des caractéristiques de la douleur par des questionnaires validés. Exploration des fibres de la nociception par test sensitif quantifié en regard de l'articulation la plus douloureuse et l'exploration de la proprioception. Résultats : 30 patients atteints du SED ont été examinés. Le sex ratio F/H était de $25 / 5$ et l'âge moyen de 24 ans. Les évènements douloureux ont débuté en moyenne à 11 ans, l'évolution vers la douleur chronique à 19 ans et le diagnostic de SED fut porté à 23 ans en moyenne, soulignant le retard diagnostique. L'évolution vers la douleur chronique fut progressive dans $3 / 4$ des cas. Des douleurs aigues, brèves et imprévisibles se sont ajoutées à un fond douloureux. La fréquence moyenne des sub-luxations était de 7 par jour, celle des luxations de 15 par an, conduisant aux urgences 9 fois par an. Petites et grosses articulations étaient douloureuses ( 7 articulations en moyenne). La douleur était d'intensité élevée (min 4,4- max 8,2/10 sur l'EVA). Une hypersensibilité cutanée fut rapportée par $50 \%$ des patients. Une allodynie au frottement fut retrouvée chez $30 \%$ des patients. En regard de l'articulation la plus douloureuse, une hypoesthésie au chaud fut constatée chez $50 \%$ des patients et le DN4 fut positif dans $3 / 4$ des cas. On retrouvait une asymétrie de la détection de la vibration sur au moins une articulation du membre inférieur chez $80 \%$ des patients. Le retentissement fonctionnel était majeur et objectivé par des scores totaux élevés au BPI (moyenne $=61 / 110$ ). La fatigue fut retrouvée chez 29/30 des patients et évaluée à 7,2/10. L'absentéisme scolaire fut évalué à 4 jours/ étaient bas malgré un taux de tentatives de suicide élevé ( $23 \%)$. Conclusion : Ce travail apporte des données nouvelles sur la douleur et son retentissement dans le SED hypermobile. Il oriente sur certains mécanismes en cause tels que les douleurs neuropathiques localisées et des phénomènes de sensibilisation centrale. Il va permettre d'ouvrir un champ de recherche pour une meilleure connaissance et reconnaissance du SED hypermobile.

Mots-clés : syndrome d'Ehlers-Danlos.

IP674. THE BURDEN OF CARDIOVASCULAR MANIFESTATIONS IN MEN AND WOMEN PRESENTING WITH FBN1 MUTATIONS

D. Detaint (1), L. Olivier-Faivre (2), A. Child (3), B.L. Loeys (4), L.C. Ades (5), E. Arbustini (6), P.N. Robinson (7), A. De Paepe (4), C. Boileau $(1,8)$, G. Jondeau (1)

(1) Centre de référence pour le syndrome de Marfan et apparentés, Hôpital Bichat, Paris, France : (2) Hôpital du Bocage, Dijon, France ; (3) Department of Cardiac and Vascular Sciences, St George's, University of London, Cranmer Terrace, London, United Kingdom ; (4) Center for Medical Genetics, Ghent University Hospital, Ghent, Belgium ; (5) Department of Clinical Genetics Marfan Research Group, The Children's Hospital at Westmead Discipline of Paediatrics and Child Health, University of Sydney, Sydney, Australia ; (6) Department of Cardiology, Fondazione IRCCS Policlinico San Matteo, Pavia, Italy ; (7) Institute for Medical Genetics, Charité-Universitätsmedizin Berlin, Berlin, Germany; (8) Hôpital Ambroise Paré, Boulogne-Billancourt, France

Contact : guillaume.jondeau@bch.aphp.fr

Background: In patients with Marfan syndrome (MFS) or other type 1 fibrillinopathy, cardiovascular (CV) involvement is the most troublesome feature. Nevertheless few data exist on its natural history and in genderrelated differences of presentation and management. Objectives: 1) To analyze CV risks and surgical management of patients with FBNI mutation; 2) To describe temporal trends over time. Methods: From a series of 1013 probands with pathogenic FBN1 mutations, data for 965 patients (median of age 22 years, male gender $53 \%$ ) without neonatal MFS were analyzed. Results: Overall the probability of ascending aortic (AA) dilatation increased throughout life, becoming almost inevitable in aldulthood (Figure). Regarding gender-related differences, women compared to men ( $\leq 40$ y: $69[99 \% \mathrm{CI}: 62-75]$ vs $79[99 \% \mathrm{CI}: 73-84], \mathrm{p}=0.0076$ ) were at lower risk of developing AA dilatation. Risks of AA dissection and prophylactic surgery were exceptional before 20 years and then increased progressively (Figure). Despite comparable risks of AA dissection ( $p=N S)$, probability of AA prophylactic surgery was significantly lower in women than in men ( $\leq 40 \mathrm{y}: 13[99 \% \mathrm{CI}: 18-20]$ vs 23[99\%Cl:17-31], $\mathrm{p}=0.0023$ ). For mitral valve (MV) involvement probability of MV prolapse and MV regurgitation increased with age whereas MV surgery remained low over time (figure). No differences were observed between gender (for all $p=N S$ ). Despite similar rate of AA dilatation over time ( $p$ for trend $=0.88$ ), rate of dissection significantly decrease from 1985 to 2000 ( $p$ for trend $=0.01$ ) Conclusion : Occurrence CV events in patients with FBN1 mutation increases throughout life. For men and women aortic monitoring is mandatory during all life. The absence of aortic dilatation should not lead to stop regular echocardiographic aortic follow-up.

Mots-clés : FBN1, marfan.

E675. ÉVALUATION MULTIDISCIPLINAIRE LONGITUDINALE DE 69 ENFANTS PORTEURS D'UNE ACHONDROPLASIE, ÉTUDE DE COHORTE

G. Baujat $\left(1^{*}\right)$, V. Cormier-Daire $\left(1^{*}\right)$, V. Topouchian $\left(2^{*}\right)$, V. Couloigner (3), J. de Blic (4), G. Finidori (2*), C. Ricour (5), T. Roujeau (6), M. Zerah (6), A. Munnich (1), M. Le Merrer $\left(1^{*}\right)$

(1) Département de Génétique Médicale (AP-HP), Hôpital Necker-Enfants Malades, Paris, France ; (2) Département d'Orthopédie Pédiatrique (AP. HP), Hôpital Necker-Enfants Malades, Paris, France ; (3) Département d'ORL Pédiatrique (AP-HP), Hôpital Necker-Enfants Malades, Paris, France ; (4) Département de Pneumologie Pédiatrique (AP-HP), Hôpital Necker-Enfants Malades, Paris, France ; (5) Nutrition Infantile (AP-HP), Hôpital Necker-Enfants Malades, Paris, France ; (6) Département de Neurochirurgie Pédiatrique (AP-HP), Hôpital Necker-Enfants Malades, Paris, France: * Centre de Référence-Maladies Osseuses Constitutionnelles Contact : genevieve.baujat@nck.aphp.fr

L'achondroplasie est une des plus fréquentes chondrodysplasies, avec une incidence estimée à $1 / 20000$ naissances. Elle est caractérisée par un déficit statural disproportionné rhizomélique, une hypoplasie de l'étage moyen de la face avec macrocrânie, des mains courtes avec doigts en trident et une hyperlordose. Cette affection est associée à des mutations du Fibroblast 
Growth Receptor 3 (FGFR3), dans $98 \%$ des cas une mutation récurrente, G1138A. Les complications sont âge-dépendantes et déterminent des schémas de prise en charge évolutifs précis. Chez l'enfant achondroplase, compression cervicomédullaire, troubles de la statique du rachis et des membres, troubles respiratoires et surcharge pondérale sont les principales préoccupations qui s'intriquent pendant les premières années de vie. Le développement cognitif est en règle normal mais certains enfants peuvent présenter un retard de langage et des difficultés d'apprentissage, récemment montrés comme secondaires aux difficultés respiratoires. Il a été également récemment publié un taux important de complications cardio-vasculaires à l'âge adulte. Nous rapportons ici l'étude rétrospective d'une cohorte de 69 sujets achondroplases, suivis depuis 15 ans de façon consécutive aux Enfants Malades, et pour lesquels ont été étudiés spécifiquement entre 0 et 15 ans : 1/ la charnière occipitale par une IRM entre 6 et 48 mois (anormale avec intervention de libération médullaire chez $30 \%$ ); $2 /$ la statique dorsolombaire (cyphose dorsolombaire nécessitant un corset chez $38 \%$ ); $3 /$ les voies aériennes supérieures (syndrome obstructif objectivé à la polysomnographie entre 1 et 6 ans chez $38 \%$ des moins de 8 ans); 4 / l'évolution des paramètres anthropométriques avec étude de l'indice de corpulence (IC) ; 5 / la qualité du langage et la réussite scolaire (difficultés modérées chez 16 sur 57 soit $28 \%$ ). Nous comparons l'évolution de l'IC avec des courbes publiées spécifiques de l'achondroplasie, et montrons des corrélations entre l'incidence des complications neurochirurgicales et respiratoires, la survenue d'une obésité et les difficultés d'apprentissage. Ce travail confirme l'importance d'un suivi médicochirurgical précoce, de la détection de la sténose de la charnière cervicale et des troubles respiratoires dont la prise en charge adaptée est garant d'une évolution cognitive normale.

Mots-clés : achondroplasie, prise en charge, cohorte.

\section{- P676. IMPACT DE LA MALADIE DE HUNTINGTON SUR L'ENTOURAGE DU PATIENT \\ C. Minet, D. Van Paemel, C. Verellen-Dumoulin, P.E. Ribaï \\ Centre de Génétique Humaine, Institut de Pathologie et de Génétique, Gosselies, Belgique \\ Contact : pascale.ribai@ipg.be}

La maladie de Huntington est une maladie neurodégénérative qui se manifeste par des signes moteurs, cognitifs et psychiatriques. Le mode de transmission autosomique dominant, l'atteinte multisystémique et le caractère évolutif de cette maladie la rendent invalidante non seulement pour le patient lui-même, mais aussi pour son entourage. Des traitements symptomatiques dans le cadre d'une prise en charge multidisciplinaire permettent d'améliorer la qualité de vie du malade et de son entourage. Cependant, peu d'études ont permis d'évaluer de manière objective l'impact de la maladie sur l'entourage du patient. Nous avons analysé les réponses de 13 accompagnants ( 10 conjoints, 2 filles et 1 sœur) de patients au questionnaire de qualité de vie de l'aidant développé en 1999 par le consortium sur l'étude de la neuroprotection et l'histoire naturelle des syndromes parkinsoniens plus (NNIPPS) et utilisé actuellement dans le réseau européen sur la maladie de Huntington. Les 13 patients étaient âgés en moyenne de 56,6 ans (45-73 ans). L'âge de début moyen de la maladie estimé par le patient était de 49,9 ans (34-63 ans), 3 patients n'ayant pas pu déterminer I'âge auquel les premiers symptômes étaient apparus. Nous avons constaté que l'accompagnant estimait l'âge de début de la maladie plus tôt que celui estimé par le patient, avec une différence moyenne de 3,5 ans (0-14 ans). Parmi les 13 patients, 5 avaient eu ou ont une addiction à l'alcool. Le score total moyen de l'échelle était de 27/96 (7-73/96), montrant de manière surprenante une qualité de vie globale de l'aidant légèrement altérée par la maladie du patient. Cependant, certaines questions de l'échelle n'étaient pas applicables pour les aidants autres que les conjoints. Nous n'avons pas trouvé de corrélation entre la dégradation de la qualité de vie de l'aidant et la sévérité de la maladie déterminée par le médecin. Nous n'avons pas non plus trouvé de corrélation entre la dégradation de la qualité de vie de l'aidant et son degré de proximité avec le patient. Nous avons analysé en détail 3 items de l'échelle et avons constaté que 10 aidants/13 avaient un sommeil perturbé avec une fatigue diurne secondaire, 6 aidants/11 rencontraient des difficultés dans leur couple et 6 aidants/12 rencontraient des difficultés professionnelles du fait de la maladie de leur parent. Des études avec un plus grand nombre de patients et un outil adapté à la maladie de Huntington sont nécessaires pour évaluer l'impact de la maladie sur la qualité de vie de l'entourage, en prenant en compte le degré de parenté mais aussi la proximité au quotidien entre l'aidant et le patient.

Mots-clés : Maladie de Huntington, entourage, aidant.

口P677. PROBLÈMES DE SANTÉ DANS UN GROUPE DE 143 ADULTES PORTEURS DE TRISOMIE 21 EN FRANCE

B. de Freminville, K. Parisi, R. Dupre Latour, F. Prieur, R. Touraine
Service de Génétique Clinique Chromosomique et Moléculaire, Centre Hospitalier et Universitaire, Saint Étienne, France Contact : benedicte.de.freminville@chu-st-etienne.fr

L'espérance de vie des personnes porteuse de trisomie 21 a considérablement augmenté ces dernières années. Cependant ces personnes présentent un risque augmenté de développer certaines pathologies qui restent souvent non diagnostiquées. Nous avons souhaité, sur un grand groupe de personnes porteuses de trisomie 21,1) évaluer leur état de santé et l'accès aux soins, 2) mesurer leurs habiletés sociales et cognitives et 3 ) retracer leur histoire médicale, éducative et rééducative. Pour cela les participants à l'étude ont été vus en consultation en présence d'un parent ou d'un éducateur. Le recueil des données s'est fait au moyen d'un questionnaire interrogeant la personne trisomique et l'accompagnant sur l'histoire médicale, les traitements, le sommeil, les problèmes de comportement (Échelle de Reiss), les capacités adaptatives (Échelle de Vineland), la présence de signes évoquant une démence (Échelle de Geydie). l'accès au système de soins, les accompagnements et prises en charge tout au long de la vie, le travail, les lieux de vie et les loisirs. Les personnes inclues dans l'étude ont eu un examen médical, des examens biologiques et ont été évaluées au moyen des Matrices progressives, colorées de Raven. Entre 2005 et 2008 nous avons inclus 143 adultes porteurs de trisomie 21, 72 femmes et 71 hommes. La moyenne d'âge est de 38,5 ans (20 à 72 ans). Plusieurs problèmes médicaux et dentaires non connus ont été détectés (hypothyroïdisme, maladie cœliaque, cataracte, surdité, apnées du sommeil, maladie d'Alzheimer,...). Nous avons aussi trouvé des difficultés d'accès aux soins pour cette population. Cette étude confirme la nécessité d'un suivi et de bilans réguliers des personnes porteuses de trisomie 21 , orientés sur la prévention des problèmes médicaux spécifiques liés à la trisomie 21 . Un dossier médical informatique, contenant les données importantes concernant la personne et partagé entre les médecins pourrait être intéressant dans le but d'améliorer la prise en charge et l'accès aux soins de cette population.

Mots-clés : Trisomie 21, suivi médical, adulte,

\section{GP678. DEVENIR PSYCHO-SOCIAL DE 13 ADULTES PORTEURS} D'UN SYNDROME DE NOONAN

J. Piard (1), M. Marvier (1), C. Beneteau (1, 2), J. Vigneron (2), H. Cavé (3), B. Leheup (1)

(1) Centre de Référence Est Syndromes Malformatifs et Anomalies du Développement, Service de Génétique Clinique CHU de Nancy et EA 4368, Faculté de Médecine Nancy-Université Université Henri Poincaré, Nancy, France: (2) Unité de Génétique, Maternité Régionale de Nancy, Nancy, France ; (3) Laboratoire de Biochimie et Génétique. Hôpital Robert Debré, Paris, France

Contact : bruno.leheup@wanadoo.fr

Le syndrome de Noonan est bien décrit dans ses aspects cliniques pédiatriques et génétiques moléculaires. Les études concernant les adultes sont plus rares. 13 adultes, porteurs d'un syndrome de Noonan avec confirmation moléculaire âgés de plus de 18 ans, 7 femmes et 6 hommes ont accepté une évaluation psychologique et ont rempli un questionnaire analysant leur parcours scolaire. Les performances cognitives ont été évaluées par la WAIS III. Le test de personnalité du Rorschach a été utilisé. L'évaluation de la qualité de vie a été réalisée par l'échelle WHOQOL-BREF, questionnaire d'auto-évaluation relatif à la santé physique, la santé psychologique, les relations sociales et l'environnement. Le questionnaire d'adaptation sociale SAS-SR (Social Adjustment Scale - Self Report) a également été administré qui évalue l'interaction entre l'individu et l'environnement social. Pour 8 des patients, le diagnostic a été porté dans l'enfance et 5 sont des parents dont l'anomalie génétique a été confirmée après diagnostic chez leur enfant. La moyenne d'âge est de 31,5 ans. La scolarité a été adaptée pour un tiers des sujets. Le dernier diplôme est un BEPC (1), un CAP ou BEP (3), un Brevet professionnel (1), un Bac pro (1), un Bac général (1) et un diplôme universitaire (3), 3 n'ont aucun diplôme. Le profil est proche de celui de la population générale de 25 à 49 ans. Le QI moyen est de 84,5 avec deux sujets présentant un retard mental (53 et 61). Le QI de Performance est de 88,4 et le QI Verbal est de 82,9 en moyenne. Le QI moyen des cas index $(77,5)$ est inférieur à celui des parents porteurs $(95,8)$. Si $70 \%$ des sujets bénéficient d'un suivi médical spécifique, aucun ne se considère comme malade. L'étude de personnalité permet de retrouver une moindre référence formelle, une relative immaturité affective et une difficulté d'imagination créatrice. Le score total au WHOQOLBREF est de 96,5 sur 120. Les patients estiment avoir une meilleure santé physique $(81,8)$ comparativement à leurs relations sociales $(78,2)$, leur santé psychologique $(76,3)$ et surtout à la qualité de leur environnement $(71,8)$. Pour le SAS-SR la note moyenne est de $1,65(1,35$ à 2,15$)$ témoignant d'une bonne adaptation sociale globale; le score moyen du critère d'adaptation au Travail est de 1,36 et celui de l'adaptation à la situation 
matérielle est de 1,15. Concernant les critères vie sociale et loisirs, vie de famille et foyer familial, les scores moyens obtenus sont nettement inférieurs, respectivement de 1,78, 1,82 et 1,77 témoignant d'une meilleure adaptation dans des domaines concrets que dans les domaines plus relationnels. La petite taille de la série ne permet pas de faire de liens avec les données moléculaires. En conclusion les données concernant cette série de patients adultes ne permettent pas de retrouver de différence significative en ce qui concerne le profil de personnalité et les résultats sont globalement bons en terme de qualité de vie et d'adaptation sociale.

Mots-clés : Noonan, adulte, devenir psycho-social.

IP680. LE RESSENTI DES DEMANDEURS D'UN DIAGNOSTIC PRÉSYMPTOMATIQUE DE LA MALADIE DE HUNTINGTON : AVANT ET APRÈS LE RÉSULTAT

M. Gargiulo (1, 2), S. Tezenas Du Montcel (3), C. Zaros (1), J. Feingold (1) A. Durr $(1,2)$

(1) AP-HP, Département de Génétique et Cytogénétique, Hôpital PitiéSalpêtrière, Paris, France; (2) Inserm U975, Université Pierre-et-MarieCurie-Paris6, Centre de Recherche de l'Institut du Cerveau et de la Moelle Épinière, Hôpital Pitié-Salpetrière, Paris, France; (3) AP-HP, Département de Biostatistique and Information médicale, Hôpital Pitié-Salpêtrière, Paris, France

Contact : alexandra.durr@upmc.fr

Le diagnostic présymptomatique (DPS) de la Maladie de Huntington (MH) est réalisé depuis 1992 dans notre centre et inclut plusieurs entretiens avec l'équipe pluridisciplinaire. Ceux-ci comportent une première phase d'information avec le généticien, suivie d'une phase de préparation avec la psychologue, et enfin de l'analyse génétique et du résultat. Notre but est de connaître le ressenti des personnes demandeuses d'un DPS avant et après le résultat. Nous avons distribué entre 1996 et 2001 un questionnaire Q1 aux consultants à l'issu de la première consultation d'information et un autre questionnaire Q2 après le résultat du DPS. 271 questionnaires Q1 ont été analysés et 152 questionnaires Q2. Ceci signifie que $60 \%$ ont abandonné leur démarche suite au premier entretien. Parmi ceux qui ont continué et obtenu un résultat, 90 étaient porteurs de l'anomalie génétique et 117 non porteurs. $37 \%$ des demandeurs déclarent que l'information génétique donnée par le généticien était différente de celle dont ils disposaient. La durée de la préparation ne leur semblait pas assez longue dans seulement $1 / 271$ avant le résultat. Par contre après le résultat, $35 \%$ estimaient que la préparation n'a pas été assez longue. Les motivations les plus citées ont été « lever le doute et savoir « $(28 \%)$, « anticiper » $(18 \%)$, "pour la descendance» $(18 \%)$ et « désir d'enfant» $(18 \%) .11 \%$ n'ont indiqué aucune motivation pour faire un DPS. La décision a été prise majoritairement $(63 \%)$ par la personne elle-même et seule. $30 \%$ seulement doutaient de leur choix. Ils pensaient que le fait d'avoir demandé un DPS ne changeait pas leur vie $(59 \%)$, et seulement $32 \%$ pensaient que leur vie changerait énormément après le résultat. Après l'annonce du résultat du test, $73 \%$ indiquaient que le résultat changeait leur vie. D'une façon intéressante, $60 \%$ indiquaient avant de poursuivre la démarche qu'ils demanderont un diagnostic prénatal en cas de résultat défavorable, sachant que seulement $16 \%$ ont réalisé ce choix. Après le résultat, les porteurs se sentent « mal « $(52 \%)$ mais $35 \%$ ont un vécu positif (versus $92 \%$ des non porteur, $\mathrm{p}<0,001) .88 \%$ des demandeurs étaient satisfaits d'avoir fait le test malgré leur résultat défavorable. $43 \%$ s'auto-observaient et guettaient les premiers symptômes de la maladie, versus $13 \%$ parmi les nonporteurs, qui continuaient à être dans le doute malgré leur résultat favorable $(p<0,05)$. Conclusion: Les consultations d'information et de préparation s'avèrent utiles car elles permettent aux demandeurs de DPS d'être mieux informés et moins banaliser l'impact que le résultat du test aura sur leur vie future. La satisfaction par rapport à leur participation, quelque soit le résultat, souligne que de vivre dans le doute sur son statut représente en soi un véritable fardeau. Il est important pour les professionnels, qui prennent en charge des demandeurs de DPS, de prendre en compte et discuter ces changements de ressenti après le résultat.

Mots-clés : diagnostic présymptomatique, maladie de Huntington, diagnostic prénatal.

DP81. EFFICACY AND SAFETY OF BEVACIZUMAB FOR THE TREATMENT OF HEMORRHAGIC HEREDITARY TELANGIECTASIA (HHT) ASSOCIATED WITH SEVERE HEPATIC VASCULAR MALFORMATIONS. PRELIMINARY RESULTS OF A PHASE II STUDY

S. Dupuis-Girod (1), I. Ginon (2), E. Guillot (3), F. Faure (4), D. Marion (5), E. Decullier (6), P.-J. Valette (3), D. Revel (7), S. Giraud (8), F. Cha-
(1) Hospices Civils de Lyon, Hôpital de l'Hôtel Dieu, Service de Génétique et centre de référence sur la maladie de Rendu-Osler, Lyon; (2) Hospices Civils de Lyon, CH Lyon Sud, Service de Cardiologie, Pierre-Bénite, France ; (3) Hospices Civils de Lyon, CH Lyon Sud, Service de Radiologie, Pierre-Bénite, France; (4) Hospices Civils de Lyon, Hôpital E. Herriot, Service d'ORL, Lyon, France : (5) Hospices Civils de Lyon, Hôpital de l'Hôtel Dieu, Service de Radiologie, Lyon, France; (6) Hospices Civils de Lyon, pôle IMER, Unité de Recherche Clinique, Lyon, France; (7) Hospices Civils de Lyon, Hôpital Louis Pradel, Service de Radiologie, Bron, France; (8) Hospices Civils de Lyon, Hôpital Édouard Herriot, Service de génétique moléculaire, Lyon, France; (9) Hospices Civils de Lyon, CH Lyon Sud, Service d'Hépato-gastroentérologie, Pierre-Bénite, France

Contact : sophie.dupuis-girod@chu-lyon.fr

Introduction : La maladie de Rendu-Osler (MRO) est une maladie vasculaire rare, de transmission autosomique dominante dont le diagnostic clinique repose sur l'association d'au moins 3 critères parmi les suivants : épistaxis, télangiectasies, caractère héréditaire, présence de malformations artério-veineuses viscérales (hépatiques, pulmonaires, cérébrales ou médullaires). Deux gènes dont les allèles mutés sont responsables de la MRO ont été identifiés et sont responsables de $90 \%$ des cas de MRO : ENG qui code pour une protéine transmembranaire appelée endogline, et ALK-I, gène de l'activine receptor like kinase de type I. La MRO serait liée à un déséquilibre de la balance " angiogénique " entre des facteurs pro-angiogénique (VEGF) et anti-angiogéniques (ALK1, BMP9). L'efficacité de traitements anti-VEGF comme le Bevacizumab a donc été envisagée dans les formes hépatiques sévères de MRO du fait des mécanismes moléculaires impliqués dans l'angiogenèse et confortée par l'amélioration de deux patients atteints de MRO avec formes hépatiques sévères responsables d'une élévation du débit cardiaque. En outre, le seul traitement curatif actuellement recommandé dans les formes hépatiques sévères de MRO est la transplantation hépatique.

Méthodologie : Il s'agit d'une étude de phase II, monocentrique. Un plan expérimental selon la méthode Gehan a été utilisé avec une première phase conçue pour éliminer rapidement un traitement inefficace et une deuxième phase permettant d'évaluer l'efficacité. Sept patients devaient être inclus dans la première étape et, selon les premiers résultats, 18 patients supplémentaires seront inclus dans la deuxième phase. Les patients inclus devaient être âgés de 18 et 70 ans, avoir un diagnostic confirmé de MRO avec une atteinte hépatique sévère responsable d'un hyperdébit cardiaque. Modalités de traitement: Le Bevacizumab est administré par voie intraveineuse à la dose de $5 \mathrm{mg} / \mathrm{kg}$ tous les 14 jours avec un total de 6 injections. Objectif principal : tester l'efficacité du Bevacizumab dans les formes hépatiques sévères de MRO. L'efficacité était définie comme une diminution de l'index cardiaque (IC), 3 mois après la première injection, par rapport à la valeur initiale au début de l'essai.

Résultats : 7 patients ont été inclus pour la première phase, parmi lesquels 2 patients n'ont pas reçu le traitement. La diminution moyenne de l'IC observée chez les 5 patients était de $9 \%$ à 3 mois avec une réponse partielle chez 3 patients (diminution moyenne de $15 \%$ ) et une non réponse chez 2 patients. Parmi les critères secondaires enregistrés, une nette amélioration des épistaxis a été observée. La tolérance du traitement a été bonne. Une seule toxicité imputable au traitement, de grade 3 a été notée (hypertension artérielle facilement équilibrée avec un traitement médicamenteux). Conclusion: Le Bevacizumab a permis une diminution de l'hyperdébit cardiaque lié aux shunts hépatiques dans la MRO à 3 mois chez $3 / 5$ des patients traités. Ceci autorise la poursuite de l'étude.

(Étude faite avec l'aide des laboratoires Roche).

Mots-clés : maladie de Rendu-Osler, Bevacizumab, shunt hépatique.

EP682. UNE INTERVENTION THÉRAPEUTIQUE ANAPLÉROTIQUE DE COURTE DURÉE AMÉLIORÉ LE MÉTABOLISME ÉNERGÉTIQUE PÉRIPHÉRIQUE DANS LA MALADIE DE HUNTINGTON

F. Mochel (1), C. Jauffret (2), S. Duteil (3), C. Marelli (1), N. Baclet (4), L. Sweetman (5), D. Rabier (6), A. Brice (1, 2), P. Carlier (3), A. Dürr (1, 2) (1) INSERM UMR S975, Hôpital de La Salpêtrière, Paris, France; (2) Département de Génétique et Cytogénétique, Hôpital de La Salpêtrière, Paris, France ; (3) Laboratoire de RMN, Institut de Myologie, Hôpital de La Salpêtrière, Paris, France; (4) Service de diététique, Hôpital de La Salpêtrière, Paris, France; (5) Institute of Metabolic Disease, Baylor Research Institute, Dallas, TX, USA ; (6) Laboratoire de Biochimie Métabolique, Hôpital Necker-Enfants Malades, Paris, France Contact : fanny.mochel@upmc.fr

A short-term anaplerotic therapeutic intervention improves peripheral energy metabolism in patients with Huntington disease. Introduction : Lors 
de l'exploration de la perte de poids aux stades précoces de la maladie de Huntington, nous avons mis en évidence une anomalie métabolique systémique suggérant un besoin de substrats énergétiques au niveau du cycle de Krebs (Mochel et al., PLoS ONE 2007). Les thérapies anaplérotiques utilisant la triheptanoïne sont développées en clinique afin de stimuler la production énergétique chez des patients présentant des déficits du cycle de Krebs à la fois au niveau central et périphérique. Par ailleurs, les métabolites de la triheptanoïne - les corps cétoniques en C5 - franchissent la barrière hémato-encéphalique. Méthodes : Nous avons mené un essai thérapeutique de courte durée - 4 jours - utilisant la triheptanoïne - $40 \%$ de 1'apport calorique total - chez 6 patients atteints de maladie de Huntington (UHDRS $=33 \pm 13,15-49$ ). Nous avons mesuré le profil des acylcarnitines et des corps cétoniques en C5 plasmatiques, ainsi que le profil urinaire des acides organiques, de façon à s'assurer de la tolérance métabolique de la triheptanoïne. Nous avons aussi analysé des marqueurs du métabolisme énergétique périphérique impliqués dans les stades précoces de la maladie de Huntington, en particulier les taux sériques bas d'IGFl et la spectroscopie RMN du phosphore 31 au niveau musculaire. Les données RMN ont été acquises au niveau de la jambe droite des patients toutes les 15 secondes pendant 15 minutes lors de la phase de récupération post exercice aérobie. Résultats: À l'état de base, la spectroscopie RMN-31P a mis en évidence une acidose musculaire post-exercice chez deux patients, responsable d'une augmentation du temps de rephosphorylation de la créatine $(\mathrm{TCr})$. A J2, le repas test utilisant la triheptanoïne a été bien toléré chez tous les patients, et en particulier, il n'y a eu aucun signe de surcharge mitochondriale par les métabolites dérivés de la triheptanoïne. Après 4 jours d'utilisation de la triheptanoïne, nous avons observé une augmentation significative des taux d'IGF1 sériques chez tous les patients (205 ng/ $\mathrm{ml} \pm 59$ versus $240 \mathrm{ng} / \mathrm{ml} \pm 83, \mathrm{p}<0,002$ ). Par ailleurs, nous avons mis en évidence une correction complète de l'acidose musculaire et du $\mathrm{TCr}$ post-exercice chez les 2 patients présentant les anomalies métaboliques avant traitement. Conclusions: Cette étude apporte une preuve de concept en faveur de l'extension de l'approche anaplérotique avec la triheptanoïne dans le traitement du déficit énergétique de la maladie de Huntington. Ce travail souligne également l'intérêt d'utiliser la spectroscopie RMN-31P pour l'étude de marqueurs du métabolisme énergétique périphérique dans la maladie de Huntington.

Mots-clés: maladie de Huntington, déficit énergétique, traitement anaplérotique.

\section{aP683. GÉNÉTIQUE ET CANCER MÉDULLAIRE DE LA THY. ROÏDE. DE LA CHIRURGIE CURATRICE À LA CHIRURGIE PROPHYLACTIQUE}

M. Boudissa (1), A. Chikouche (2), M. Ait Abdellah (2), L. Griene (2), Y. Oukaci (2), M. Semrouni (1)

(1) Service d'endocrinologie, EHS Centre Pierre-et-Marie-Curie(CPMC), Alger, Algérie ; (2) Laboratoire d'hormonologie, Unité de biologie Moléculaire CPMC, Alger, Algérie

\section{Contact : mebarek.boudissa@yahoo.fr}

Introduction. Le CMT est une tumeur neuroendocrine des cellules parafolliculaires de la thyroïde. Il représente 3,95 à $6,35 \%$ des cancers thyrödiens en Algérie. Il est soit sporadique dans $75 \%$, ou familial dans $25 \%$ des cas, transmis sur le mode autosomique dominant. Le gène responsable du CMT est le proto-oncogène RET, marqueur génétique spécifique. But du travail. L'analyse systématique du gène RET devant tout nouveau cas de CMT au CPMC, à la recherche de formes familiales, afin de permettre une prise en charge précoce (voir dans certains cas prophylactique) des cas apparentés génétiquement à risque. Matériel et méthode. 38 CMT ont bénéficié d'une étude génétique, qui a permis de retrouver 7 formes familiales $(18,42 \%)$. L'enquête génétique chez 4 des 7 familles a retrouvé : 2 cas dans une famille (A) mutation $768,-3$ cas dans la $2^{e}$ famille (B) mutation 634. Une patiente apparentée de la famille B a refusé toute exploration. - Aucun dans les familles $\mathrm{C}$ et $\mathrm{D}$. - Pour trois familles l'enquête est en cours. 2 de la famille A et 2 de la famille B, ont bénéficié d'une thyroïdectomie totale (TT1) et l'étude histologique a conclu : - chez la famille A : - Une thyroïde saine des deux cas, - Chez la famille B : - Un micro cancer de $2 \mathrm{~mm}$ avec hyperplasie des cellules $\mathrm{C}$ ( $\mathrm{HCC}$ ) multifocale et bilatérale dans un cas - Une hyperplasie multifocale et bilatérale chez l'autre. La guérison a été chez les 4 cas, confirmée par un test à la pentagastrine postopératoire normal. Conclusion. À travers ce modeste travail qui montre que grâce aux données du dépistage génétique nouvellement introduit en Algérie nous sommes passés de la chirurgie curatrice à la chirurgie prophylactique.
@ P684. LES DÉFICITS EN BIOTINIDASES : INTÉRÊT DU DAGNOSTIC PRÉCOCE

A. Mekki, K. Haddad, A. Lebied

Service de Pédiatrie, CHU Parnet, H. Dey Alger, Algérie

Contact:dr.lebied@yahoo.fr

Introduction Le déficit en biotinidase est une maladie génétique liée à une mutation du gène BTD situé sur le chromosome 3 en P25, transmise sur le mode autosomal récessif et responsable d'une perturbation des carboxylases. Les principaux symptômes apparaissent au cours des premiers jours de vie comprennent des signes cutanés, des manifestations neurologiques associées à une acidose lactique. Non traitée la maladie aboutit à la mort ou à des séquelles neurologiques. Objectif L'objectif de l'étude est de dégager les principaux signes cliniques et biochimiques de la maladie pour un diagnostic précoce aisé. Patients et méthodes À partir d'une série rétrospective de 08 cas hospitalisés, entre Janvier 2002 et Décembre 2007. Les nourrissons atteints 05 garçons et 03 filles étaient âgés de 01 à 08 mois au moment de leur hospitalisation. 06 cas présentaient des signes cutanés et neurologiques, des lésions cutanées pour 02 cas. Tous les malades ont bénéficié d'un bilan métabolique (gazométrie, lactates, pyruvates, et ammoniemie) avec EEG, FO et imagerie cérébrale. 07 malades d'une étude enzymatique (la Biotinidase sérique), 07 PEA ont été réalisés. 01 malade est décédé d'une infection intercurrente. Le recul moyen est de 3 ans. Discussion et résultats Notre étude comportait 08 cas dont 03 observés au sein d'une même fratrie. L'âge moyen au diagnostic était de 3,6 mois avec une légère prédominance masculine. Les signes cutanés étaient inauguraux dans les 08 cas $(100 \%)$ : conjonctivite bilatérale dans 08 cas $(100 \%)$ associée à une dermatose chez 06 malades $(75 \%$ des cas) et une alopécie avec perte des sourcils dans 04 cas $(50 \%)$. Les manifestations neurologiques apparaissent après un intervalle libre dans $100 \%$ des cas avec hypotonie, épilepsie myoclonique dans les 06 cas et coma dans 04 cas. L'activité biotinidase était effondrée voire nulle chez 07 malades. 07 malades ont bénéficié d'un traitement par la biotine à raison de $10 \mathrm{mg} / \mathrm{j} 04$ enfants présentent un retard mental dont 02 sévères. 04 PEA étaient pathologiques avec surdité bilatérale grave dans 02 cas. Conclusion II ressort de notre étude que devant l'association de signes cutanés et d'une encéphalopathie progressive, le diagnostic de déficit en biotnidase est évoqué,il doit être est confirmé par l'étude de l'activité de la biotinidase effondrée. C'est une encéphalopathie métabolique « traitable ». Le traitement remarquablement efficace repose sur l'administration à vie de biotine. Le pronostic est donc étroitement lié à la précocité du diagnostic pour prévenir les séquelles. Le dépistage systématique de cette affection est désormais possible.

Mots-clés : déficit en caboxylase, acidose métabolique, sines cutanés, alopecie.

IP685. PRISE EN CHARGE DE L'HYPODONTIE D'ENFANTS ATTEINTS DE DYSPLASIE ECTODERMIQUE

D. Favoli, C. Georges, C. Phillip, C. Tardieu

Centre de compétence pour le traitement des manifestations odontologiques des maladies rares, Service d'odontologie, Hôpital Timone, Marseille, France

Contact : corinne.tardieu@ap-hm.fr

Les dysplasies ectodermiques sont des maladies rares affectant sévèrement les organes issus de l'ectoderme fætal, comme les dents. Elles entraînent donc des anomalies de l'émail dentaire (dérivé ectodermique), de la dentine et d'une partie du squelette maxillo-facial (dérivés mésodermiques). La fréquence des dysplasies ectodermiques est estimée à 1 à 7 pour 100000 naissances toutes formes confondues. Des anomalies dentaires telles que les agénésies sont fréquemment notées puisqu'elles sont recensées dans 111 formes sur environ 160 décrites. L'hypodontie dentaire génère notamment des troubles du développement oro facial, un retard d'acquisition des praxies oro faciales et une perturbation de l'esthétique du visage. Dans le cadre de la consultation d'odontologie pédiatrique à l'hôpital de la Timone, les enfants sont pris en charge dès l'age de 2 ans. Afin d'améliorer la prise en charge, un protocole de prise en charge est proposé selon les âges (denture temporaire, mixte ou permanente), la sévérité de l'hypodontie (oligontie, anodontie, d'une ou deux arcades) et selon les pathologies ou anomalies dentaires associées à l'hypodontie (anomalie d'éruption, anomalie de forme, dysharmonie dento-dentaire, carie, sécheresse buccale, dyspraxie linguale). Les propositions thérapeutiques techniquement possibles sont décrites telles que la réhabilitation prothétique, l'orthodontie, la restauration esthétique, l'orthophonie. Elles n'ont pas été toujours acceptées par l'enfant. Une discussion est ouverte sur les limites liées à la bonne compréhension du plan de traitement par l'enfant dont les attentes ne sont pas en adéquation parfaite avec celles de ses parents.

Mots-clés : dysplasies ectodermiques, agénésie, dentition. 
DP86. COMPLICATION ET TRAITEMENT DES PATIENTS BÊTA-THALASSÉMIQUES EN FRANCE: RÉSULTATS DU REGISTRE NATIONAL

I. Thuret $(1,2)$, C. Pondarré $(2,3)$, M.C. Siméoni (4), C. Badens $(2,5)$ et les cliniciens de la fédération des centres de référence et de compétence "Pathologie de l'érythrocyte "

(1) Service d'hématologie pédiatrique, Hôpital d'enfants de la Timone, Marseille, France; (2) Centre de référence Thalassémie, Hôpital d'enfants de la Timone, Marseille, France ; (3) Institut d'Hématologie et d'Oncologie Pédiatrique, Lyon, France; (4) Unité d'aide méthodologique à la Recherche Clinique et Epidémiologique, Faculté de Médecine, Marseille, France ; (5) Laboratoire de Génétique Moléculaire, Département de génétique, Hôpital d'enfants de la Timone, Marseille, France Contact : catherine.badens@ap-hm.fr

Dans le cadre du plan Maladies Rares, un registre national de patients thalassémiques a été développé par le centre de référence des Thalassémies. Des données épidémiologiques et cliniques ont été collectées pour les patients atteints de forme majeure ou intermédiaire de bêta-thalassémies résidents en France. Le recueil a été effectué par l'envoi d'une fiche d'inclusion à tous les cliniciens en charge de patients thalassémiques et participant aux différents réseaux nationaux. D'autres sources ont été sollicitées : Société Française de Greffe de Moëlle, réseau des laboratoires de diagnostic moléculaire des hémoglobinopathiés, laboratoires de dépistage néonatal. Une mise à jour était demandée tous les 18 mois. Une étude transversale réalisée en Février 2009 retrouve 378 patients inclus dont 267 avec une forme majeure. Leur médiane d'age est de 20 ans. Les patients sont répartis dans 57 centres dont 31 pédiatriques. $70 \%$ des patients sont nés en France. L'incidence de la forme majeure de la maladie est estimée à 1 pour 100000 . Après ajustement sur l'age, la croissance staturale, la proportion de patients adultes ayant eu au moins un enfant, le taux de splénectomie et de cholécystectomie ne sont pas significativement différents chez les patients thalassémiques majeurs non greffés et ceux atteints de thalassémie intermédiaire. Ces résultats reflètent les progrès du traitement conventionnel de la thalassémie majeure mais aussi un recrutement préférentiel dans le registre, des patients thalassémiques intermédiaires les plus sévères. L'évaluation de la surcharge en fer par IRM cardiaque n'a été pratiquée que pour $40 \%$ des patients de plus de 10 ans. De 2005 à 2008 , un changement radical dans la répartition des traitements chélateurs du fer a été observé : la Deferoxamine, administrée en perfusion lente sous-cutanée, était administrée à $75 \%$ des patients chélatés en 2005 alors que le Déférasirox, actif par voie orale, devenait à partir de 2007 le traitement le plus fréquemment prescrit. La fréquence des complications et les valeurs de la ferritinémie observées dans le registre national sont très proches de celles rapportées dans les autres pays d'Europe ou en Amérique du Nord. L'estimation de la surcharge tissulaire cardiaque par IRM est, en revanche, moins répandue en France qu'en Italie ou au Royaume-Uni. La mise en place de ce registre a, d'ores et déjà, permis de stimuler la pratique des examens complémentaires, de diffuser les recommandations les plus récentes, de resserrer les liens entre les cliniciens et le centre de référence. Le suivi longitudinal des patients permettra, dans le futur, de disposer de données sur la mortalité au sein de la cohorte française et d'évaluer l'effet direct du registre sur la prise en charge clinique des patients thalassémiques en France.

Mots-clés : thalassémies, registre.

-P687. MALADIE DE WOLMAN, OU LA NÉCESSITÉ VITALE DU DIAGNOSTIC PRÉCOCE D'UNE MALADIE LYSOSOMIALE

E. Hullo (1), K. Mention (2), V. Valayannopoulos (2), A. Pagnier (1), C. Bost-Bru (1), F. Dufernez (3), M.T. Vanier (4), T. Levade (5), D. Plantaz (1), P. de Lonlay (2), P. Benlian (3)

(1) Clinique Universitaire de Pédiatrie, CHU Grenoble, France: (2) Centre de Référence sur les Maladies Rares du Métabolisme, hôpital Necker, APHP-Paris, France ; (3) Laboratoire de Référence pour le Diagnostic des Maladies Rares, hôpital Saint Antoine, APHP-Paris, France ; (4) Laboratoire Gillet Merieux, Centre Hospitalier Lyon Sud, France ; (5) Laboratoire de Biochimie Métabolique, CHU Rangueil, Toulouse, France Contact : pascale.benlian@sat.aphp.fr

Contexte. La maladie de Wolman est une maladie autosomique récessive exceptionnelle ( $<50$ cas décrits) qui résulte du déficit complet en lipase acide lysosomiale (LIPA). Le défaut de dégradation lysosomiale des lipides neutres, engendre l'accumulation massive et généralisée de stérols et de triglycérides, à l'origine d'un décès $<6-12$ mois de vie, par dénutrition, inflammation et défaillance hépatique (IHC) majeures, alors même que les fonctions cardiaques et neurosensorielles sont normales. La caractérisation du gène et de la structure cristallographique de l'enzyme, ont offert depuis n'existe pas d'autre traitement à l'heure actuelle, que de restaurer au moins partiellement l'activité enzymatique de la LIPA, compatible avec une survie normale. Seule la greffe de moelle osseuse (GMO) s'est avérée efficace dans 3 cas publiés sur 5 . Objectifs. Si la nécessité du diagnostic semble établie, l'âge critique auquel un traitement salvateur est encore possible, reste mal connu. Nous rapportons notre expérience sur 4 cas de maladie de Wolman diagnostiqués depuis 2004 par une double approche biochimique et moléculaire. Résultats. Dans tous les cas, les signes d'appels sont des vomissements sans diarrhée, survenant entre 1 et 3 semaines de vie, associés à une hépatosplénomégalie pas ou peu fébrile et une cassure de la courbe pondérale. La calcification bilatérale des surrénales plus souvent visible à l'échographie, ou par TDM du fait d'un météorisme important dès l'âge de 1 mois, est quasi pathognomonique ; une adénomégalie abdominale est notable. La biologie est marquée par une cholestase non ictérique, une cytolyse, une hypertriglycéridémie modérée $(<3,00 \mathrm{~g} / \mathrm{l})$ avec cholestérol total normal, un syndrome inflammatoire, et la présence de leucocytes vacuolés spumeux. Une consanguinité parentale était connue dans 3 cas. Les éléments du diagnostic et l'évolution clinique sont présentés ci-après. Age / État au Diagnostic : Activité/Génotype LIPA : Evolution/ Suivi. Cas 1: 3 mois/ IHC, Dénutrition : $0,01 \mathrm{xN}$ /G321W (HMZ): Décès : 4,5 mois. Cas 2: 3 mois/IHC, Dénutrition : Ind./R44X (HTZ)+D331VfsX47 (HTZ) : Décès : 3,5 mois, DPN ensuite à 12 SA (Enfant sain, HTZ). Cas 3:2 mois/Cytolyse, Malabsorption: Ind./Épissage (c. 429-1 G>C, HMZ) : GMO : 3 mois Ambulatoire : 6 mois, Chimérisme : $3 \%$ receveur, LIPA $=\mathrm{N}$, Fonctions Hépatiques $=\mathrm{N}$. Cas $4:$ 2 mois/Cytolyse : 0,06 xN/ W119S (HMZ) : GMO : 2,5 mois/En cours. Conclusion. Diagnostiqué avant 2 mois de vie, le déficit complet en LIPA, même partiellement corrigé par une GMO avant l'âge de 3 mois, permet de voir se restaurer rapidement les fonctions digestives et hépatiques, et d'envisager une survie sans séquelles neuromusculaires, bien au-delà de l'espérance de vie «naturelle » de la Maladie de Wolman.

Mots-clés : lipase acide lysosomiale, maladie de wolman, maladie lysosomiale.

\section{IP688. INTÉRÊT DU TRAITEMENT PAR ARGININE DANS LES SYNDROMES LEIGH/MELAS ASSOCIÉS À DES MUTATIONS DANS LES GENES ND3 ET ND5}

A. Chaussenot (1), S. Bannwarth (1, 2), K. Fragaki (1, 2), C. Rouzier (1, 2), B. Funalot (3), G. Auge (1), B. Chabrol (4), V. Paquis-Flucklinger (1, 2)

(1) Service de génétique médicale, Hôpital Archet 2, CHU de Nice, France: (2) IGMRC, FRE CNRS / UNSA 3086, Université de Nice Sophia-Antipolis, France ; (3) Service de Neurologie, Hôpital Dupuytren, CHU de Limoges, France ; (4) Service de Neurologie pédiatrique, Hôpital Timone, $\mathrm{CHU}$ de Marseille, France

\section{Contact : chaussenot.a@chu-nice.fr}

Des études récentes ont suggéré l'efficacité du traitement par Arginine sur les épisodes de « pseudo-stroke » dans les MELAS (Mitochondrial Encephalomyopathy Lactic, Acidosis and Stroke-like episodes) liés à la mutation 3243 tRNALeu, en perfusion à forte dose lors de la phase aiguë, mais également par voie orale au long cours, en diminuant de manière significative la fréquence et la sévérité de ces épisodes. Nous rapportons 2 observations d'évolution favorable sous Arginine de patients avec un tableau clinique de chevauchement de type Leigh/MELAS avec des mutations m.10191T>C et m.13513G >A, identifiées par la technique Surveyor. Il s'agit de 2 garçons âgés de 7 et 17 ans ayant présenté plusieurs épisodes déficitaires avec une récupération partielle parfois complète évoquant des "pseudo-strokes ", avant de développer une régression prolongée sévère avec atteinte des noyaux gris centraux, évoquant un syndrome de Leigh. L'hypothèse de la présence de ces 2 mécanismes physiopathologiques était confirmée par les données des IRM et du PET-scan. Le traitement par Arginine a été introduit lors de la régression alors que le pronostic était très péjoratif. Après avoir observé une période de stabilisation, ces 2 enfants ont présenté une amélioration. L'absence de nouvelles lésions sur l'IRM de suivi a permis de conforter cette observation. Les 2 mutations identifiées, m.10191T $>\mathrm{C}$ et $\mathrm{m} .13513 \mathrm{G}>\mathrm{A}$, ont été fréquemment associées à des syndromes de Leigh et parfois des MELAS dans la littérature. L'Arginine, précurseur de l'acide nitrique, agirait principalement en favorisant la microvascularisation cérébrale (en préservant les cellules endothéliales et en augmentant le débit sanguin) et en diminuant les lésions médiées par les radicaux libres. Dans les «pseudo-strokes » du MELAS, il existe une altération segmentaire de la vasodilatation des artères intracérébrales et une production d'espèces réactives oxygénées (ROS), ce qui permet de comprendre en partie l'efficacité de l'Arginine. Ces deux observations montrent que l'Arginine pourrait également être efficace dans les tableaux de type Leigh/Melas. Nous suggérons d'élargir les indications 
thérapeutiques de l'Arginine aux tableaux cliniques de type Leigh/MELAS et nous soulignons l'importance d'identifier des épisodes déficitaires lors de syndromes de Leigh à début tardif pouvant relever d'un mécanisme de type " pseudo-stroke ». De même, il pourrait être intéressant d'étudier son action sur les syndromes de Leigh en se basant sur la diminution des lésions liées aux radicaux libres observée avec l'Arginine.

Mots-clés : arginine, syndrome de Leigh, MELAS.

\section{P689. SURCHARGES EN FER : PART RELATIVE DES FACTEURS} GÉNÉTIQUES ET DES FACTEURS ENVIRONNEMENTAUX

V. Scotet (1, 2), C. Berquier (2), M.M. Mérour (2), A.Y. Mercier (2), B. Chanu (2), I. Gourlaouen (2), G. Le Gac $(1,2)$, C. Férec $(1,2)$ (1) Inserm, U613, Brest, France ; (2) Établissement Français du SangBretagne, site de Brest, France

Contact : virginie.scotet@univ-brest.fr

Objectif : L'hémochromatose héréditaire de type I (liée au gène HFE) est une maladie génétique complexe particulièrement fréquente dans les populations d'Europe du Nord. C'est l'une des causes de surcharge en fer dans l'organisme. L'objectif de cette étude était donc de mieux connaître la population des patients adressés pour soustractions sanguines dans le cadre d'une surcharge en fer dans un centre de santé de l'Établissement Français du Sang, et de déterminer la part des patients présentant une hémochromatose génétique. Méthode : L'étude incluait tous les patients présentant une hyperferritinémie pris en charge par saignées entre le 01/01/2004 et le 31/12/2007 dans le centre de santé brestois de l'EFS-Bretagne, où la prévalence de l'hémochromatose héréditaire de type I est particulièrement élevée. Un questionnaire clinique, complété par les médecins référents, renseignait sur le sexe, l'âge au diagnostic, les habitudes de vie, les signes biologiques et cliniques. Nous avons pu déterminer les fréquences génotypiques, puis mesurer le poids des facteurs environnementaux chez les patients ne présentant pas le génotype majoritaire C282Y/C282Y. Résultats : Sur la période 2004-2007, 326 patients ont été inclus dans un protocole de saignées, $68,1 \%$ étant des hommes. Sur le plan génotypique, seuls $56,0 \%$ des patients portaient le génotype C282Y/C282Y. Près de $20 \%$ des patients étaient C282Y/H63D (19,2\% - génotype 5 fois plus fréquent que dans la population générale, $\mathrm{p}<0,0001)$ tandis que $7,2 \%$ des patients ne portaient ni la mutation $\mathrm{C} 282 \mathrm{Y}$, ni le facteur de susceptibilité H63D. Le poids des facteurs environnementaux était significativement plus important chez les patients non $\mathrm{C} 282 \mathrm{Y} / \mathrm{C} 282 \mathrm{Y}$ et était inversement corrélé au nombre de mutations dans le génotype. Ainsi, une consommation excessive d'alcool était observée chez 10,3\% des patients C282Y/C282Y, 21,6\% des patients $\mathrm{C} 282 \mathrm{Y} / \mathrm{H} 63 \mathrm{D}$ et $31,6 \%$ des patients $\%(\mathrm{p}=0,0017)$. Des résultats similaires étaient observés pour le surpoids $(39,4 \%, 67,3 \%$ et $72,7 \%$ respectivement), qui apparaissait ainsi quatre fois plus fréquent chez les patients $-/(\mathrm{OR}=4,1[1,4-12,5], p=0,0031)$ et trois fois plus fréquent chez les patients C282Y/H63D (OR $=3,2[1,6-6,4], p=0,0003)$ que chez les $\mathrm{C} 282 \mathrm{Y} / \mathrm{C} 282 \mathrm{Y}$. Ces résultats demeuraient inchangés après ajustement sur le sexe et l'âge. Discussion: Cette étude révèle que seule un peu plus de la moitié des patients pris en charge par saignées présente une hémochromatose génétique définie par l'homozygotie C282Y. Cette étude rappelle qu'il est indispensable de réaliser un diagnostic d'exclusion des formes principales de surcharges en fer secondaires (syndrome dysmétabolique, syndrome inflammatoire, consommation excessive d'alcool, cytolyse) avant d'engager une étude génétique.

Mots-clés : hémochromatose héréditaire, surcharge en fer, gène HFE.

\section{IP690. APPLICATION ET EFFICACITÉ DU SAUT D'EXON INDUIT PAR OLIGONUCLEOTIDES ANTISENS DANS LES DYSFERLINOPATHIES}

N. Wein (1), A. Avril (2), M. Bartoli (1), S. Chaouch (2), P. Laforet (3), A. Behin (3), G. Butler-Browne (2), V. Mouly (2), M. Krahn (1, 4), L. Garcia (2), N. Lévy $(1,4)$

(1) Université de la Méditerranée, Inserm UMR_S 910 * Génétique Médicale et Génomique Fonctionnelle », Faculté de Médecine de Marseille, France ; (2) Université Pierre-et-Marie-Curie, Paris VI, Inserm UMR_S 974, CNRS UMR 7215, Institut de Myologie, Groupe Hospitalier PitiéSalpêtrière, Paris, France ; (3) Centre de référence de pathologie neuromusculaire Paris Est, Groupe Hospitalier Pitié-Salpêtrière, AP-HP, Paris, France ; (4) Département de Génétique Médicale, Hôpital d'enfants de la Timone, AP-HM, Marseille, France

Contact : nicolas.levy@univmed.fr

Les mutations dans le gène codant la dysferline (DYSF, Chr. 2p13 55 exons, mRNA $6,2 \mathrm{~kb}$ ) causent différents phénotypes, principalement la dystrophie musculaire des ceintures de type 2B (Limb Girdle Muscular Dystrophy type 2B, LGMD2B) et la myopathie de Miyoshi (MM), de transmission autosomique récessive. Ces deux myopathies ont en commun un début chez l'adulte jeune, une évolution lente de la maladie, mais pouvant évoluer vers une perte de la marche, et une élévation importante du taux de CPK. Des phénotypes intermédiaires ont été décrits sous le terme de distal limb girdle dystrophy, avec un déficit à la fois proximal et distal. La dysferline (2080 acides aminés, $237 \mathrm{kDa}$ ) comporte 7 domaines $\mathrm{C} 2$, impliqués dans la liaison $\mathrm{Ca} 2+$ et un domaine transmembranaire $\mathrm{C}$-terminal. À ce jour, la prise en charge des patients est uniquement symptomatique puisqu'il n'existe aucune approche thérapeutique spécifique. En raison de la similarité des domaines $\mathrm{C} 2$ qui composent la dysferline, une stratégie par saut d'exon pourrait être envisagée. Cependant contrairement à la dystrophie musculaire de Duchenne, très peu de délétions associées à un phénotype atténué ont été répertoriées. Seul deux cas cliniques ont pu mettre en évidence une délétion d'une partie de la protéine associée à un phénotype atténué. En particulier, l'identification d'une délétion en phase de l'exon 32 du gène DYSF chez une patiente présentant une forme clinique très modérée de dysferlinopathie nous a conduit à considérer cet exon comme cible potentielle pour une approche thérapeutique par saut d'exon. Nous avons identifié par analyse informatique trois sites essentiels au maintien de l'exon 32. Ces sites ont été ciblés par transfection d'oligonucleotides antisens ou par incorporation dans des lentivirus codant ces antisens. Les expériences ont été réalisées sur des myoblastes dérivés de fibroblastes, issus de témoins et de deux patients porteurs de mutations dans l'exon 32. Parmi les trois cibles testées, deux ont permis de mettre en évidence un saut d'exon efficace de l'exon 32 à la fois sur les cellules de patients et de témoins. Étant donné le rôle démontré de la dysferline dans la réparation de la membrane du sarcolemme, des tests fonctionnels visant à évaluer le gain de fonction après traitement ont été réalisés. Ces résultats ont montré que cette delta32-dysferline est capable de réparer les membranes des myotubes déficients après lésion. II semble donc que l'absence de l'exon 32 ne soit pas néfaste à la fonction de la dysferline. Ceci constitue un argument important pour le caractère non essentiel de cet exon, et la propriété en partie modulaire de la dysferline. Cette preuve de principe naturelle démontre qu'une stratégie par saut d'exon est envisageable pour des patients comportant des mutations dans l'exon $32 \mathrm{du}$ gène de la dysferlin dont 13 à ce jour sont répertoriés dans la base UMD-Dysf).

Mots-clés : dysferline, saut d'exon, therapie.

DP691. ÉTABLISSEMENT D'UNE BASE DE DONNÉES FRANCPAISE UMD-SMA

P. Saugier-Veber (1, 2), B. Gerard (3), V. Humbertclaude (4), J.M. Cuisset (5), C. Andre (6), D. Hamroun (4), T. Frebourg (1, 2), C. Beroud (4) (1) Inserm U614, Faculté de Médecine et de Pharmacie, Institut Hospitalo-Universitaire, Université de Rouen, France: (2) Service de Génétique, CHU de Rouen, France; (3) Laboratoire de Biochimie Génétique, Hôpital Robert-Debré, Paris, France ; (4) Laboratoire de Génétique Moléculaire, Institut Universitaire de Recherche Clinique, Montpellier, France ; (5) Service de neuropédiatrie, CHRU de Lille, France ; (6) AFMdirection scientifique, Évry, France

Contact:Pascale.Saugier-Veber@chu-rouen.fr

L'amyotrophie spinale infantile (SMA) est caractérisée par une dégénérescence progressive des motoneurones de la corne antérieure de la moelle épinière, responsable de paralysies avec amyotrophie et dénervation. Avec une incidence de $1 / 6000$, la SMÀ représente la deuxième maladie autosomique récessive létale après la mucoviscidose. Les patients sont classés en trois groupes, type I, II ou III, en fonction de l'âge de début de l'affection et de son évolution. La SMA résulte de l'inactivation à l'état homozygote du gène SMN1 en 5q11-q13. À ce jour, tous les essais thérapeutiques se sont soldés par des échecs. À l'heure actuelle, il n'existe pas de base de données à l'échelle nationale intégrant des données cliniques et moléculaires sur la SMA. Les trois objectifs principaux pour la base de données SMA sont : 1 - collecter des données épidémiologiques sur la SMA en France, 2 - promouvoir les projets de recherche clinique (corrélations génotype/phénotype pour l'identification de gènes modificateurs, histoire naturelle de la SMA), 3 - faciliter les essais thérapeutiques. La base de données SMA contiendra des données cliniques et moléculaires. Les items cliniques ont été sélectionnés pour obtenir une synthèse du statut clinique du patient à un instant $\mathrm{T}$. Chaque item est défini à 3 niveaux : symptôme, sévérité et âge. Les items moléculaires comprennent le type de mutation du gène SMN1 (délétions, mutations ponctuelles) et le nombre de copies des gènes SMN1 et SMN2. Les données cliniques seront implémentées et réactualisées chaque année par les curateurs cliniques à partir des Centres de Référence Neuromusculaires. Les items moléculaires seront validés (et complétés si nécessaire) par les curateurs moléculaires. Les données, pseudo-anonymisées, seront saisies dans l'outil UMD hébergé 
dans l'unité INSERM U827 à Montpellier, avec le consentement du patient. L'outil UMD-SMA sera également utilisé dans d'autres pays, permettant le transfert d'une partie des données vers le registre global de TREATNMD, regroupant les données des registres SMA de plusieurs pays. L'équipe INSERM U827 de Montpellier est responsable du développement de ce registre global et du transfert des données. L'objectif de ce registre global de TREAT-NMD est de faciliter l'inclusion des patients dans les futurs essais thérapeutiques internationaux. Une collaboration étroite avec les généticiens moléculaires et les neurologues/neuropédiatres est nécessaire pour la réalisation de cette base de données française UMD-SMA. Cette base de données sera particulièrement utile pour la réalisation des essais thérapeutiques, tant en amont de l'essai pour évaluer sa faisabilité en comptant le nombre de patients qui pourraient potentiellement être inclus, que pour l'essai lui-même en facilitant le transfert d'informations vers le neurologue/neuropédiatre pour la proposition d'inclusion de leur patient.

Mots-clés: amyotrophie spinale infantile, base de données, essais thérapeutiques.

\section{P692. CILIARY BEATING RECOVERY IN DEFICIENT HUMAN AIRWAY EPITHELIAL CELLS AFTER LENTIVIRUS EX VIVO GENE THERAPY}

B. Chhin (1), D. Negre (2), O. Merrot (3), J. Pham (1), Y. Tourneur (4, 5), D. Ressnikoff (5), M. Jaspers (6), M. Jorissen (6), F.L. Cosset (2), P. Bouvagnet $(1,7)$

(1) EA 4171, Université de Lyon, France: (2) Université de Lyon, (UCBLyonI), IFR128; INSERM, U758; Ecole Normale Supérieure de Lyon, Lyon, France; (3) Service ORL, Hospices Civils de Lyon, Hôpital de la Croix-Rousse, Lyon, France ; (4) INSERM, UMR886, Cardioprotection; (5) Centre Commun de Quantimétrie (CCQ), Université de Lyon, Lyon, France : (6) ENT department, Head and Neck Surgery, UZ GHB, Leuven, Belgium; (7) Laboratoire Cardiogénétique, Hospices Civils de Lyon, Groupe Hospitalier Est, Bron, France

Contact : Patrice.Bouvagnet@recherche.univ-lyonl.fr

Primary Ciliary Dyskinesia is a heterogeneous genetic disease which is characterized by cilia dysfunction of the epithelial cells lining the respiratory tracts resulting in recurrent respiratory tract infections. Despite lifelong physiological therapy and antibiotics, the lungs of affected patients are progressively destroyed leading to respiratory insufficiency. Recessive mutations in Dynein Axonemal Intermediate chain type 1 (DNAI1) gene have been described in 10\% of cases of Primary Ciliary Dyskinesia. Our goal was to restore normal ciliary beating in DNAII deficient human airway epithelial cells. A lentiviral vector based on Simian Immunodeficiency Virus pseudotyped with Vesicular Stomatitis Virus Glycoprotein was used to transduce cultured human airway epithelial cells with a cDNA of DNAIl driven by the Elongation Factor 1 promoter. Transcription and translation of the transduced gene were tested by RT-PCR and western blot, respectively. Human airway epithelial cells which were DNAIl deficient due to compound heterozygous mutations and consequently had immotile cilia and no outer dynein arm, were transduced by the lentivirus. Cilia beating was recorded and electron microscopy of the cilia was performed. Transcription and translation of the transduced DNAIl gene were detected in human cells treated with the lentivirus. In addition, immotile cilia recovered a normal beat and outer dynein arms reappeared. We demonstrated that it is possible to obtain a normalization of ciliary beat frequency of deficient human airway epithelial cells by using a lentivirus to transduce cells with the therapeutic gene. This preliminary step constitutes a conceptual proof which is indispensable in the perspective of Primary Ciliary Dyskinesia's in vivo gene therapy. This is the first time that recovery of cilia beating is demonstrated in this disease.

Mots-clés : gene therapy, lentivirus, Cilia.

EP693. DIRECTED INTEGRATION OF NEW INSULATED LENTIVIRAL VECTORS TO THE HETEROCHROMATIN TOWARDS SAFER GENE TRANSFER TO STEM CELLS

A. Artus (1), C. Duros (1), Y. Botbol (2), S. Schultz (3), M. Schmidt (3), C. von Kalle (3), M. Lavigne (2), O. Cohen-Haguenauer (1, 4)

(1) LBPAV CLINIGENE, Ecole Normale Superieure (ENSC), Cachan, France; (2) Laboratory of Structural Virology, Pasteur Institute, Paris, France ; (3) Laboratory of Translational Oncology, Deutsches Krebsforschungszentrum (DKFZ), Heidelberg, Germany ; (4) Hôpital Saint-Louis, Paris, France

Contact : odile.cohen@ens-cachan.fr

The need for better gene transfer systems towards improved risk/benefit balance for patients remains a major challenge in the clinical translation of gene therapy (GT). We have investigated the improvement of integrating vectors safety in combining (i) new short synthetic genetic insulator elements (GIE) and (ii) directing genetic integration to heterochromatin. In otherwise successful GT-trials in SCID patients and others, insertional mutagenesis has resulted in leukemia from transduced cells. The identification of new GIEs which would prevent such activation effects is a main challenge since attempts e.g. with the chicken beta-globin HS4, have met with poor efficacy and genetic instability. We have constructed SIN-insulated retrovectors with two candidate GIEs and compared them to native and SIN-LTRs. With each constructs two internal promoters have been tested : either the strong Fr-MuLV-U3 or the housekeeping hPGK. We could identify a specific combination of insulator 2 repeats which translates into best functional activity, high titers and boundary effect in both gammaretro (p20) and lentivectors (DCaro4). In target cells a dramatic shif of expression is observed with an homogenous profile the level of which is strictly conditioned by the promoter strength. These data remain stable in both HeLa cells over three months and cord blood HSCs for two months, irrespective of the multiplicity of infection (MOI). In comparison, control vectors show heterogeneous expression profiles with levels which depend on the MOI and prove unstable. We have undertaken genotoxicity assessment in comparing integration patterns ingenuity in human target cells sampled over three months using high-throughput pyro-sequencing. Preliminary data indicate a flat pattern without CIS. Since GIEs are believed to shield the transgenic cassette from inhibitory effects and silencing, DCaro4 has been further tested with chimeric HIV-1 derived integrases which comprise C-ter chromodomains targeting heterochromatin through either histone H3 (ML6 chimera) or methylated CpG islands (ML10). With DCaro4 only and both chimeras, a homogeneous expression is evidenced in over $20 \%$ of the cells which is sustained over time. With control lentivectors, less than $2 \%$ of cells express GFP as compared to background using a control double-mutant in both catalytic and ledgf binding-sites ; in addition, a two-times increase of expression can be induced with histone deacetylase inhibitors. Our approach could significantly reduce integration into open chromatin sensitive sites in stem cells at the time of transduction, a feature which might significantly decrease subsequent genotoxicity, according to X-SCIDs patients data. This work has been performed with the support of the EC-DG research within the FP6-Network of Excellence, CLINIGENE: LSHB-CT-2006-018933.

Mots-clés : thérapie génique, mutagenèse insertionnelle, intégration ciblée. 